\title{
The metabolic roots of senescence: mechanisms and opportunities for intervention
}

\author{
Christopher D. Wiley $\circledast^{1,2} \bowtie$ and Judith Campisi ${ }^{2,3}$
}

\begin{abstract}
Cellular senescence entails a permanent proliferative arrest, coupled to multiple phenotypic changes. Among these changes is the release of numerous biologically active molecules collectively known as the senescence-associated secretory phenotype, or SASP. A growing body of literature indicates that both senescence and the SASP are sensitive to cellular and organismal metabolic states, which in turn can drive phenotypes associated with metabolic dysfunction. Here, we review the current literature linking senescence and metabolism, with an eye toward findings at the cellular level, including both metabolic inducers of senescence and alterations in cellular metabolism associated with senescence. Additionally, we consider how interventions that target either metabolism or senescent cells might influence each other and mitigate some of the pro-aging effects of cellular senescence. We conclude that the most effective interventions will likely break a degenerative feedback cycle by which cellular senescence promotes metabolic diseases, which in turn promote senescence.
\end{abstract}

Historic perspective on cellular senescence. Cellular senescence, the non-dividing altered state into which many vertebrate cells enter when stressed, was first formally described by Hayflick and Moorhead in the early 1960s (refs. ${ }^{1,2}$ ). Their finding-that normal cells eventually cease dividing (in culture)-challenged the long-held idea initiated by Alexis Carrel in the early 1900s that normal cells were intrinsically 'immortal' (in culture) ${ }^{3}$. And, whereas Carrel suggested that organismal mortality might be a consequence of multi-cellularity, Hayflick and Moorhead suggested that the eventual cessation of cell division (subsequently termed cellular senescence) reflected organismal aging. They also noted that cells derived from malignant tumors did not undergo this form of senescence, suggesting that the senescent state existed to suppress the development of cancer. Many years later, Sager and colleagues showed that cellular senescence was a response to potential tumor-inducing stimuli, and thus formally postulated that the senescence response was a potent anti-cancer mechanism ${ }^{4,5}$. Since the 1960 s, our understanding of cellular senescence, including its physiological and pathological roles, has exploded. In addition, it is now apparent that senescent cells both experience and cause many aspects of metabolic reprogramming, some aspects of which we discuss below.

Cell autonomous and non-autonomous effects of senescent cells. At the core of the cellular senescence response is an essentially irreversible arrest of cell proliferation (growth). It is now clear-from multiple lines of evidence-that this arrest constitutes a potent, cell autonomous anti-cancer mechanism in vivo ${ }^{6,7}$. Consequently, most humans and mice harboring mutations that prevent or suppress the senescence growth arrest (for example, p53 or pRB inactivating or dominant negative mutations $\left.s^{8,9}\right)$ are cancer-prone and generally die an early death due to malignant tumors.

In addition, certain senescent cells become moderately resistant to cell death. In some senescent cells, this cell autonomous resistance is due to increased expression of anti-apoptotic, compared to pro-apoptotic, proteins, a characteristic shared by several cancer cells. This property has led to the repurposing of certain (mostly failed) anti-cancer drugs that were initially designed to kill tumor cells, but now are being tested as senolytics-that is, drugs that can selectively kill senescent cells, but not non-senescent counterparts ${ }^{10-12}$. However, it is not yet clear how pervasive cell death resistance is among different senescent cell types. For example, senescent endothelial cells are in general more prone to spontaneous apoptosls than senescent fibroblasts, although even fibroblasts can undergo apoptosis following senescence when faced with certain types of DNA damage (for example, transcription-blocking lesions $)^{13}$. In any case, the mechanisms of cell death resistance in senescent cells remains incompletely understood.

Finally, all senescent cells thus far examined develop a complex, multi-component senescent-associated secretory phenotype (SASP). The SASP acts cell non-autonomously to alter the behavior of neighboring cells and the tissue microenvironment. The SASP is strikingly variable and plastic. It depends on the cell type and senescence inducer (discussed below), and is dynamic, changing characteristics over time ${ }^{14,15}$. A striking, but not sole, feature of the SASP is the preponderance of pro-inflammatory molecules, including cytokines, chemokines, bioactive lipids and damage-associated molecular patterns (DAMPs; also termed Alarmins). Chronic inflammation is, of course, a major risk factor for many age-related diseases, including late-life cancer; hence the term 'inflammaging' has been used to describe the chronic inflammation that is a common attribute of aged tissues and might be at least partially due to the accumulation of senescent cells in aged tissues ${ }^{16-18}$.

In recent years, it has become apparent that the phenotypes of senescent cells, in addition to the SASP, are remarkably variable, heterogeneous and plastic ${ }^{19}$. For example, for many-but not all ${ }^{20}$ senescent cells, the growth arrest is initiated and/or enforced by the cyclin-dependent kinase and cell cycle inhibitors $\mathrm{p} 16^{\mathrm{INK} 4 \mathrm{a}}$ and $\mathrm{p} 21^{\mathrm{CIP} 1}$ (encoded by the CDKN2A and CDKN1A genes). Likewise, other widely used senescence markers, such as the senescence-associated $\beta$-galactosidase (SA- $\beta$ gal) ${ }^{21}$, reduced expression of the nuclear lamina protein lamin B1 (LMNB1) ${ }^{22}$, and the relocalization and secretion of the nuclear protein HMGB1 (where, following secretion, it acts as a DAMP $)^{23}$, are neither invariable nor universal senescence markers. Thus, assessing the ability of specific stimuli to induce a

'Jean Mayer USDA Human Nutrition Research Center on Aging at Tufts University, Boston, CA, USA. ${ }^{2}$ Buck Institute for Research on Aging, Novato, CA, USA. ${ }^{3}$ Lawrence Berkeley National Laboratory, Berkeley, CA, USA. $₫ e$-mail: Christopher.Wiley@tufts.edu 
senescence response-and assessing the burden of senescent cells in tissues in vivo-requires the use of multiple markers ${ }^{24}$.

Adaptive vs maladaptive effects of senescent cells. The senescence response can be beneficial or deleterious, depending on the physiological context. This dualism is consistent with the evolutionary theory of antagonistic pleiotropy. Antagonistic pleiotropy postulates that traits selected to ensure the survival of young organisms in natural environments, in which life spans are short, can become deleterious in modern protected environments, in which life spans are significantly longer ${ }^{25}$. Thus, aging is likely a consequence of the declining force of natural selection with age.

With regard to the beneficial effects of cellular senescence, as noted above, the senescence growth arrest protects young organisms from developing cancer. In addition, SASP factors can optimize the morphogenesis of certain structures in the embryo ${ }^{26,27}$, and initiate parturition in the placenta ${ }^{28,29}$. Finally, senescent cells occur transiently at sites of tissue damage where they contribute to wound healing, tissue repair and regeneration, most likely through specific SASP factors ${ }^{30-34}$.

In contrast, senescent cells increase with age in most mammalian tissues, where they appear to persist. Whether this increase is due to increased production or decreased clearance, for example by the immune system, is unclear. More importantly, experiments using human cells and tissues, transgenic mouse models and pharmacological interventions in cells and mice strongly implicate senescent cells in a large number of age-related pathologies, ranging from neurodegeneration to, ironically, age-related cancer ${ }^{6,35-38}$. Most of the detrimental effects of senescent cells can be attributed to the SASP, which, as noted above, is rich in pro-inflammatory molecules.

What initiates a senescence response? Little is known about how senescent cells are induced in vivo, particularly during aging. Known inducers of senescence responses-at least in cultured cells and mouse models-include, among others, DNA damage, activated oncogenes and mitochondrial dysfunction (discussed below). Because senescent cells are rare, even in old and diseased tissue, it has been difficult to determine how they were induced to senesce in vivo. However, single-cell profiling, at both the trancriptomic and proteomic levels, promises to help identify the major drivers of senescence during natural aging and in age-related pathologies. In all cases, senescent cells must undergo metabolic reprogramming in order to maintain their viable growth-arrested state and express the genes and proteins needed to sustain the highly complex, dynamic and heterogenous $\mathrm{SASP}^{14,39}$. The causes and consequences of these metabolic shifts are discussed below.

\section{Metabolic drivers of senescence}

Several forms of metabolic stress can both drive senescence and influence the SASP (Fig. 1). Here we describe some of these and discuss them in the context of aging and disease.

Mitochondrial dysfunction. Mitochondria are major regulators of age-related pathology. Mice that accumulate mitochondrial DNA (mtDNA) mutations at an accelerated rate age prematurely ${ }^{40,41}$, whereas overexpression of mitochondrially-targeted catalase (mCAT) preserves mitochondrial function and extends lifespan in mice ${ }^{42}$. It is therefore not surprising that senescence and the SASP are similarly responsive to the function of mitochondria within the cell.

Many drivers of mitochondrial dysfunction also result in cellular senescence. These drivers include mtDNA depletion and mutations, inhibitors of the electron transport chain, loss of the mitochondrial chaperone protein HSPA9, loss of the mitochondrial protein deacetylase SIRT3 and the de-acylase SIRT5 ${ }^{43}$, and disruptions in complex I assembly ${ }^{44}$. This mitochondrial dysfunction-associated senescence (MiDAS) phenotype lacks several of the proinflammatory parts of the SASP, but instead includes its own distinct set of SASP factors. MiDAS is primarily driven by the accumulation of cytosolic NADH, which is normally oxidized by the mitochondria to $\mathrm{NAD}^{+}$(Fig. 2). This lowering of the $\mathrm{NAD}^{+} / \mathrm{NADH}$ ratio inhibits the key glycolytic enzyme, GAPDH, resulting in ATP depletion, AMPK activation and cell cycle arrest ${ }^{43}$.

Additionally, mitochondria are a source of reactive oxygen species (ROS). Loss of mitochondrial superoxide dismutase (SOD2) in mice drives cellular senescence ${ }^{45}$. A transgenic cell culture model of mitochondrial elimination by mitophagy protects against senescence and the expression of many pro-inflammatory SASP factors; this protection is associated with reductions in both ROS and nuclear DNA damage foci, especially telomere-associated foci ${ }^{46}$. Similarly, mitochondrial ROS can activate the JUN N-terminal kinase, which in turn promotes release of cytosolic chromatin fragments and activation of proinflammatory components of the SASP ${ }^{47}$. These and many other studies suggest that mitochondria-derived ROS may be a key driver of cellular senescence.

Notably, despite evidence that senescent cells drive aging and limit both health span and median lifespan ${ }^{48,49}$, few interventions that directly antagonize ROS extend lifespan ${ }^{50}$. Moreover, none of these interventions have demonstrated a reduction in age-related accumulation of senescent cells. Rather, studies that target mitochondria and prevent senescence or SASP modules in vivo also target other aspects of mitochondrial biology. Thus, it is an open question as to whether mitochondrial ROS are actually causal for the accumulation of senescent cells with age. For example, PGC-1B depletion lowers mitochondrial biogenesis, and rapamycin increases mitophagy ${ }^{46}$. Although both manipulations decrease ROS, they also alter many other aspects of mitochondrial metabolism and function. Additionally, ROS are essential signaling molecules for several processes, such as cell proliferation ${ }^{51}$, innate immunity ${ }^{52}$, stem cell renewal ${ }^{53,54}$, differentiation ${ }^{55}$ and survival ${ }^{56}$. The relationship between ROS, senescence and aging in vivo is therefore likely to be more complex than culture models currently indicate.

More recent studies also showed that lowering endoplasmic reticulum (ER)-mitochondria contact by ablating the Itpr 2 calcium channel in mice lowers the number of age-related senescent cells and the levels of several SASP factors, resulting in an extension of health span and lifespan ${ }^{57}$. However, it is unclear if these effects are due to reduced mitochondrial calcium overload, diminished ER-mitochondrial contacts, or some combination of both. These strategies demonstrate a definitive role for mitochondria in the age-related accumulation of senescent cells, but are not specifically informative about whether ROS, per se, are to blame. The role mitochondrial function in cellular senescence and its associated phenotypes is also the subject of several recent reviews ${ }^{58-61}$.

An important caveat to many cell culture studies, especially those using mouse cells, is that while ROS-including mitochondrial ROS - can certainly induce senescence in culture, a majority of these studies were performed in atmospheric, rather than physiological, oxygen concentrations, which alters senescence responses ${ }^{62}$. Thus, many of the observed phenomena are potential artifacts of a hyperoxic state. Next, we discuss the role of oxygen levels in the development of senescence.

Oxygen. Phenotypes of cellular senescence are highly dependent on the levels of oxygen available to the cell. This dependence is often inversely correlated, as more oxygen tends to accelerate senescence. With over 60 oxygen-consuming enzymes in the mammalian genome $^{63}$, it is not surprising that oxygen levels are a key modulator of many biological processes, including senescence. Murine cells are more sensitive to oxygen levels, as murine fibroblasts cultured at atmospheric oxygen levels undergo rapid senescence, but, due to endogenous telomerase activity, will proliferate extensively if oxygen levels are lowered to 3\%-closer to levels observed in the stroma 

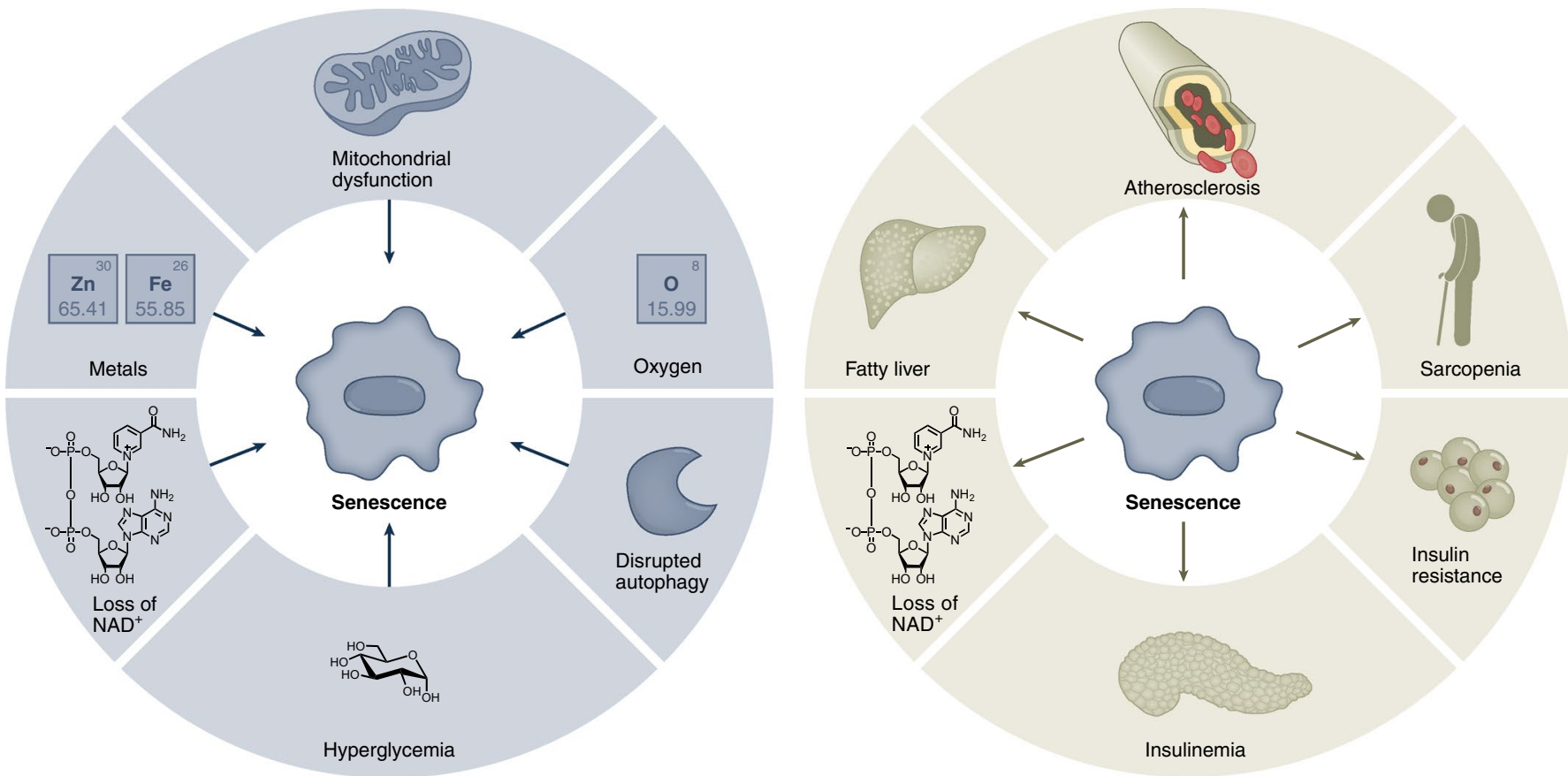

Fig. 1 | Relationships between metabolism and cellular senescence. Left: Metabolic drivers of senescence. Mitochondrial dysfunction can drive senescence through disruption of cytosolic $\mathrm{NAD}^{+} / \mathrm{NADH}$ ratios, production of reactive oxygen species, and potentially other mechanisms. Accumulation of excess metals (especially transition metals) also promotes senescence. Loss of NAD+ results in senescence through loss of sirtuin or PARP activities and changes in cellular redox states. Hyperglycemia can drive senescence, although mechanistic detail is still needed. Disrupted autophagy can drive senescence in some contexts, but also prevent it in others. Non-physiological oxygen levels influence the development of senescent cells, with higher oxygen generally favoring senescence. Right: Senescent cells as drivers of metabolic disease. Senescent cells and/or the SASP can drive both formation of atherosclerotic plaques as well as plaque instability. In the liver, senescent cells can promote steatosis. The SASP also activates macrophages, which elevate CD38 and lower tissue NAD+ evels. In the pancreas, senescent $\beta$-cells promote hyperinsulinemia, but as $\beta$-cells are attacked by the immune system, this can become hypoinsulinemia. In peripheral tissues (for example, fat) senescent cells can promote insulin resistance-so senescent cells can drive diabetes and metabolic disease in multiple ways. Finally, senescent cells promote sarcopenia in muscle tissue, which can influence basal metabolism, activity levels, and frailty.

in vivo ${ }^{62}$. Extension of replicative lifespan and stemness are also observed in a subset of human cell lines, such as human amniotic fluid stem cells ${ }^{64}$. Additionally, atmospheric $\mathrm{O}_{2}$ levels are required for senescence reinforcement following treatment with drugs that activate cell cycle arrest, such as nutlin-3a ${ }^{65,66}$. Furthermore, lower $\mathrm{O}_{2}$ levels are required for a human-like SASP in cultured murine fibroblasts ${ }^{67}$, and this SASP is closer to that observed with age. Despite these findings, sub-physiological levels of oxygen (that is, hypoxia) can also activate AMPK, which can suppress the SASP by mTOR inhibition ${ }^{68}$. More broadly, these data signal the potential for artifacts when studying senescence (and likely many other conditions) using atmospheric rather than physiological oxygen levels. Oxygen concentrations vary strongly depending on the tissue and species $^{69}$, so physiological levels for relevant cell types is an important consideration when studying senescence. Several recent articles review the role of oxygen in diverse biological phenomena, including senescence ${ }^{63,69-71}$.

Unfortunately, hyperoxia is a medical necessity under certain conditions. For example, premature infants, whose lungs have not yet developed, often require supplemental oxygen but later experience increased risks of respiratory conditions, such as asthma, in early childhood ${ }^{72}$. Similarly, individuals that experience the severe symptoms of COVID-19 often require supplemental oxygen ${ }^{73}$. Treatment of fetal human airway smooth muscle cells and lung fibroblasts with moderate hyperoxia $\left(40 \% \mathrm{O}_{2}\right)$ increased markers of senescence in as short as 7 days ${ }^{72,74}$, suggesting that senescent cells may play a role in these disorders. Conversely, hyperbaric $100 \%$ oxygen was recently found to lower markers of senescence in specific populations of peripheral blood mononuclear cells ${ }^{75}$, so cell type may play an important role in senescent cell responses to oxygen. Additionally, a murine cardiac ischemia-reperfusion model accumulates senescent cells, and elimination of these cells improves recover ${ }^{76}$, indicating that fluctuations in oxygen can also drive senescence and pathology. Oxygen level is therefore an important consideration both in cell culture settings, and potentially for the formation of senescence under specific circumstances in animal models and humans.

Disrupted NAD ${ }^{+}$metabolism. $\mathrm{NAD}^{+}$plays a major role in the regulation of both the cell cycle arrest and the SASP during senescence (Fig. 2). NAD ${ }^{+}$is a major cofactor for both poly-ADP-ribose polymerase (PARP) and sirtuin family proteins (SIRTs). PARP antagonizes senescence by protecting against genotoxic stress ${ }^{77}$, but also promotes NF-kB activation and secretory phenotypes in senescent cells $^{78}$. Indeed, loss of PARP1 during epithelial cell senescence is associated with increased levels of unrepaired single strand breaks, which in turn reinforce cell cycle arrest through p38MAPK activation $^{79}$. Similarly, inhibition of nicotinamide phosphoribosyltransferase (NAMPT) - which prevents NAD salvage and lowers total $\mathrm{NAD}^{+}$levels-both promotes a senescence arrest and suppresses proinflammatory aspects of the $\mathrm{SASP}^{43,80}$; this effect resembles the phenotype of MiDAS. Importantly, $\mathrm{NAD}^{+}$levels decline with age in many tissues, and this decline is associated with multiple degenerative conditions, including age-related muscle loss and diabetes ${ }^{81-83}$.

Several sirtuins play key roles in senescence response and the SASP. Beyond SIRT3 and SIRT5, which antagonize MiDAS ${ }^{43}$, SIRTs 


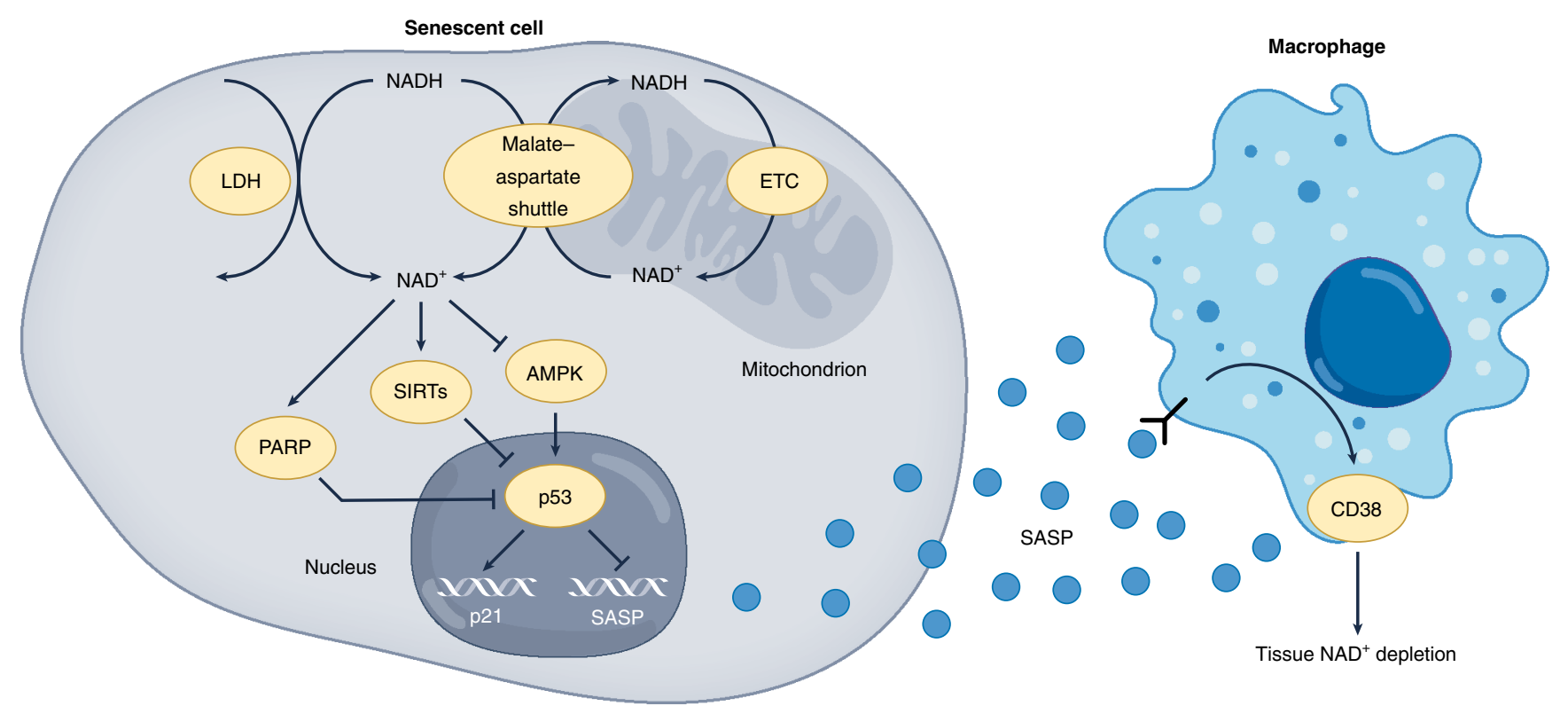

Fig. 2 | NAD Metabolism and Cellular Senescence. $N A D^{+}$levels and NAD + NADH ratios are controlled by multiple pathways during senescence. The $\mathrm{NAD}^{+} / \mathrm{NADH}$ ratio is maintained by conversion of pyruvate to lactate in the cytosol, or by transfer of reducing equivalent of NADH to the mitochondrion by the malate-aspartate shuttle. NADH is then oxidized back to $\mathrm{NAD}^{+}$in the mitochondrion by the activity of Complex I of the electron transport chain (ETC). Disruption of any of these processes leads to increased cytosolic NADH, AMPK activation, and senescence. NAD+ acts through PARPs and sirtuins to prevent genotoxic stress and p53 activation, which promotes senescence, but antagonizes the SASP. Once released by senescent cells, SASP factors can bind their cognate receptors on macrophages, which then elevate CD38. CD38 then lowers tissue levels of NAD+ in the surrounding tissue as part of cyclic ADP ribose (cADPR) synthesis.

1, 2 and 6 have known roles in senescence. SIRT2 deacetylates and stabilizes the mitotic checkpoint kinase BUBR1, which declines during aging ${ }^{84}$, and protects against aneuploidy-a potent driver of senescence ${ }^{85}$. Notably, supplementation with the $\mathrm{NAD}^{+}$precursor nicotinamide mononucleotide (NMN) extends the median lifespan of progeric BUBR1 hypomorphic mice ${ }^{86}$. SIRT6 knockout mice show premature aging and a hyperinflammatory state that is driven at least in part by de-repression of retrotransposons ${ }^{87}$, which also occurs with senescence ${ }^{88}$. Furthermore, overexpression of SIRT6 prevents the loss of homologous recombination observed during senescence $^{89}$. Finally, SIRT1 is degraded by autophagy during senescence $^{90}$. Loss of SIRT1 activity is associated with multiple aspects of senescence, including SASP activation ${ }^{91}$ and cell cycle arrest ${ }^{92}$, as well as several senescence-associated degenerative pathologies including neurodegeneration, cachexia, fatty liver and atherosclero$\operatorname{sis}^{93,94}$. Together, the sirtuins and PARP demonstrate the importance of $\mathrm{NAD}^{+}$-consuming enzymes in senescence and help explain why loss of $\mathrm{NAD}^{+}$levels with age can be so deleterious to a tissue.

$\mathrm{NAD}^{+}$is not always beneficial in the context of senescence. We hypothesized that since loss of $\mathrm{NAD}^{+}$antagonizes the SASP, increased $\mathrm{NAD}^{+}$might have the opposite effect and promote segments of the SASP ${ }^{39}$. This proved to be the case as NAMPT and $\mathrm{NAD}^{+}$are elevated in senescent cells. This rise is associated with decreased AMPK and p53 activation, leading to increased p38MAPK and NF-kB activity and the secretion of proinflammatory SASP factors ${ }^{80}$. Thus, $\mathrm{NAD}^{+}$may antagonize senescence, but once senescence is established, $\mathrm{NAD}^{+}$increases may instead promote degenerative pathologies through the SASP.

Cellular senescence also plays a role in tissue-level $\mathrm{NAD}^{+}$metabolism. The SASP promotes CD38 activation in macrophages. CD38 consumes $\mathrm{NAD}^{+}$during the synthesis of cyclic-ADP ribose $(\mathrm{cADPR})^{95,96}$, and is a primary driver of $\mathrm{NAD}^{+}$depletion with age ${ }^{97}$. Therefore, senescent cells may drive $\mathrm{NAD}^{+}$loss, which in turn could lead to more senescent cells (Fig. 1). This degenerative feedback loop could therefore drive multiple $\mathrm{NAD}^{+}$and senescence-dependent age-related conditions (for example, diabetes ${ }^{82}$ ). In contrast, eliminating senescent cells or suppressing the SASP might protect $\mathrm{NAD}^{+}$homeostasis, and $\mathrm{CD} 38$ inhibition in turn might be protective and prevent increased senescence with age.

As noted above, MiDAS results from lowered cytosolic $\mathrm{NAD}^{+}$/ $\mathrm{NADH}$ ratios ${ }^{43}$, but reduced $\mathrm{NAD}^{+} / \mathrm{NADH}$ ratios can occur without mitochondrial dysfunction. For example, inhibition of the malate-aspartate shuttle, which transports reducing equivalents of NADH from the cytosol to the mitochondria, by aminooxyacetate (AOA) drives senescence ${ }^{43}$. Loss of the shuttle enzyme malate dehydrogenase 1 (MDH1) has a similar phenotype ${ }^{98}$. Similar results occur when malic enzymes, which catalyze $\mathrm{NAD}^{+}$-dependent conversion malate to pyruvate in the cytosol (ME1) and mitochondria (ME2) are inhibited ${ }^{99}$. These enzymes are reciprocally regulated by $\mathrm{p} 53$; depletion of either drives p53-dependent senescence, but p53 in turn represses malic enzymes ${ }^{99}$. These studies show that the interplay between $\mathrm{NAD}^{+} / \mathrm{NADH}$ ratios and $\mathrm{p} 53$ is a key regulator of senescence under conditions of metabolic stress.

Hyperglycemia. Numerous studies demonstrate that culturing cells in high levels of glucose accelerates cellular senescence ${ }^{100,101}$. However, because multiple pathways by which this acceleration occurs have been identified, no unifying model currently explains all forms of hyperglycemia-associated senescence. Most studies focus on endothelial cells, including retinal endothelial cells, but fibroblasts and renal epithelial cells have also been studied ${ }^{100-104}$. Nonetheless, the few mechanisms ascribed to senescence induction seem unlikely to be common to multiple cell lineages. For example, arginase 1 (ARG1) expression and activity are induced in retinal endothelial cells cultured in high glucose, resulting in reduced nitric oxide (NO) and elevated oxidative stress ${ }^{101}$. Retinal endothelial cells in a mouse model of streptozotocin (STZ)-induced diabetic retinopathy undergo senescence in an ARG1-dependent manner ${ }^{105}$, and inhibition of ARG1 activity prevents high glucose-induced senescence in cultured retinal epithelial cells and diabetes-induced 
retinal senescence in mice. Ironically, prior reports in human umbilical vein endothelial cells (HUVECs) showed that arginase activity declined during high-glucose-induced senescence, and this decline was linked to decreased NO synthesis ${ }^{106}$. Supplementation with arginine prevented these effects and antagonized senescence in high-glucose-treated HUVECs. Generally, NO antagonizes endothelial cell senescence ${ }^{106}$, so the key effector of glucose-induced senescence in these cells is likely related to the level of cellular NO production. This mechanism is unlikely to be universal because ARG1 is expressed in only a few cell types, and NO is not produced at appreciable levels by all cells.

High glucose also drives senescence in fibroblasts ${ }^{100,104}$, and glucose restriction can extend replicative lifespan ${ }^{107}$. Although fibroblasts produce NO, they do not express ARG1, so it is unclear whether the phenotypes observed with endothelial cells translate to this cell type. For example, inhibition of nitric oxide synthases (NOS) with $\mathrm{N}(\mathrm{G})$-nitro-L-arginine methyl ester (L-NAME) drives senescence in endothelial cells ${ }^{106}$, but can antagonize senescence when fibroblasts are co-cultured with macrophages ${ }^{108}$. Thus, the role of $\mathrm{NO}$ in the senescence responses of different cell types requires further investigation.

In another study, high glucose promoted senescence in fibroblasts by lowering the levels of SIRT3, and transgenic overexpression of SIRT3 prevented this high glucose-induced senescence ${ }^{104}$. Loss of SIRT3 can drive MiDAS ${ }^{43}$, so lowering of SIRT3 by excess glucose is an appealing link between these processes. Furthermore, high glucose accelerates the loss of sirtuin expression in endothelial cells ${ }^{103}$, and this loss is also associated with increased senescence. Since SIRT3 deacetylates mitochondrial proteins, and high-sugar diets can increase mitochondrial protein acetylation ${ }^{109,110}$ and decrease $\mathrm{NAD}^{+} / \mathrm{NADH}$ ratios ${ }^{11}$, it is possible that hyperglycemia drives senescence by MiDAS or a MiDAS-like phenotype.

Hyperglycemia can also result in the formation of advanced glycation end-products (AGEs), which can drive senescence in some models. For example, treatment of kidney proximal tubule epithelial cells with exogenous AGEs (for example, glycated albumin) triggered increased expression of p21 (ref. ${ }^{112}$ ) or p16 (ref. ${ }^{113}$ ), coupled to activation of SA- $\beta$ gal, in a receptor for advanced glycation end-products receptor (RAGE)-dependent manner. Notably, these studies focused only on effects observed 48 hours after treatment, so chronic exposure and effects of AGE removal were not considered. Further, these effects may not extend to other cell types. Conversely, activation of RAGE extends the replicative lifespan of murine preadipocytes by binding to p53 and antagonizing p 21 expression ${ }^{114}$. More research is therefore required to fully elucidate the relationship between AGEs and cellular senescence. Furthermore, studies linking high glucose to senescence have not addressed the SASP, and therefore the consequences of this form of senescence are still somewhat unclear. Despite these needs, the links between diabetes and senescence are growing, and increases in senescent cells at sites of diabetic complications indicate that this is an important area for future research.

\section{Metabolism of senescent cells}

Senescent cells show several alterations in their metabolic states (Fig. 3). Here we review some of these alterations and their significance in the development of senescence phenotypes.

Lipid metabolism. A growing body of evidence indicates that senescent cells alter lipid metabolism. One of the first observations along these lines came from the finding that the sphingomyelin-ceramide pathway, and especially sphingomyelinase, was significantly more active in senescent cells ${ }^{115}$. Addition of ceramides at concentrations that approximate those found in senescent cells lowers markers of cell proliferation and increases markers of senescence in both fibroblasts $^{115}$ and endothelial cells ${ }^{116}$. Overall, ceramide and sphingosine promote senescence, whereas sphingosine-1-phosphate (S1P) promotes cell proliferation via its cognate receptors. Ceramide-activated protein phosphatases (PP1 and PP2A) can promote a senescence arrest by dephosphorylating CDK2 and specifically up-regulating p21 (ref. ${ }^{117}$ ). Sphingosine has similar effects, but the mechanisms are less understood ${ }^{118}$. The relationships among ceramides, sphingolipids and cellular senescence were recently reviewed ${ }^{119}$.

Additional studies indicate that senescent cells upregulate $\beta$-oxidation-the metabolic breakdown of lipids ${ }^{120}$-and that this activity is necessary for the secretion of many proinflammatory components of the SASP in oncogene-induced senescence. In addition, fatty acid synthase (FASN) was elevated in senescent cells and required for both the cell cycle arrest and parts of the SASP ${ }^{121}$. The expression of several enzymes related to both fatty acid synthesis (for example FASN) and oxidation (for example ACADS, ACADL, ACADSB, ACADVL) increase in senescent cells ${ }^{122}$, whereas stearoyl-CoA desaturase-which converts saturated fatty acids into monounsaturated fatty acids (MUFAs) - declines ${ }^{123}$. These data suggest that saturated fatty acids might be an important substrate for energy production in senescent cells.

Beyond energy use and storage, fatty acids play additional roles in senescence responses. Notably, lipid droplets increase and accumulate with at least some forms of cellular senescence. Exogenous lipids are preferentially incorporated into triacylglyerols, which form the lipid droplets in senescent cells ${ }^{123}$. Furthermore, free polyunsaturated fatty acid (PUFA) levels increase in senescent cells ${ }^{124}$, likely via p38-dependent activation of phospholipase A2, which cleaves PUFAs from cellular membranes ${ }^{125}$. Senescent cells incorporate higher levels of PUFAs into triglycerides ${ }^{126}$, which ultimately accumulate in lipid droplets. Notably, animal models of obesity show increased numbers of senescent cells in the brain, and these cells show increased numbers of lipid droplets. Importantly, clearance of senescent cells lowered the accumulation of these droplets ${ }^{127}$. Lowering exogenous lipid levels in cell culture media prevented both lipid droplet accumulation and the upregulation of many SASP factors $^{127}$. These data suggest that lipid droplets, or at least the presence of lipids, is required for some key aspects of cellular senescence and the SASP.

The release of free PUFAs from the plasma membrane is associated with multiple forms of senescence ${ }^{124,126}$. Additionally, levels of several PUFAs, such as eicosapentanoic acid (EPA), arachidonic acid (AA), dihomo- $\gamma$-linolenic acid (DGLA) and docosohexanoic acid (DHA), are highly elevated during $\mathrm{MiDAS}^{124}$, above other forms of senescence. This elevation may reflect an attempt by cells to preserve the NAD redox balance by using PUFA desaturation to convert NADH into $\mathrm{NAD}^{+}$, as described ${ }^{128}$. Beyond accumulating as triglycerides in lipid droplets, free PUFAs are also converted to oxygenated signaling molecules collectively known as oxylipins ${ }^{124}$. Oxylipins associated with cellular senescence include leukotrienes ${ }^{125,129}$ and prostaglandins, including dihomo-prostaglandins ${ }^{124,130}$. Senescence-associated leukotriene release drives profibrotic features of pulmonary fibrosis, and eliminating senescent cells ameliorates these effects ${ }^{125,131}$. The synthesis of prostaglandins is essential for reinforcing the senescence-associated cell cycle arrest, as inhibitors of the inducible prostaglandin synthase 2 (PTGS2 or COX-2) extend the replicative lifespan of cultured cells ${ }^{132}$ and allow increased numbers of dividing cells in response to genotoxic stress-induced and oncogene-induced senescence ${ }^{124}$.

Surprisingly, it does not appear that prostaglandin synthesis promotes senescence through interactions with prostaglandin receptors. Rather, senescent cells upregulate expression of the prostaglandin transporter (PGT/SLCO2A1), but without a commensurate elevation in expression of prostaglandin dehydrogenase (PGDH), which inactivates prostaglandins by converting them to 15 -keto derivatives. These metabolic changes result in the intracellular accumulation of 15-deoxy prostaglandins, especially 15d-PGJ2 


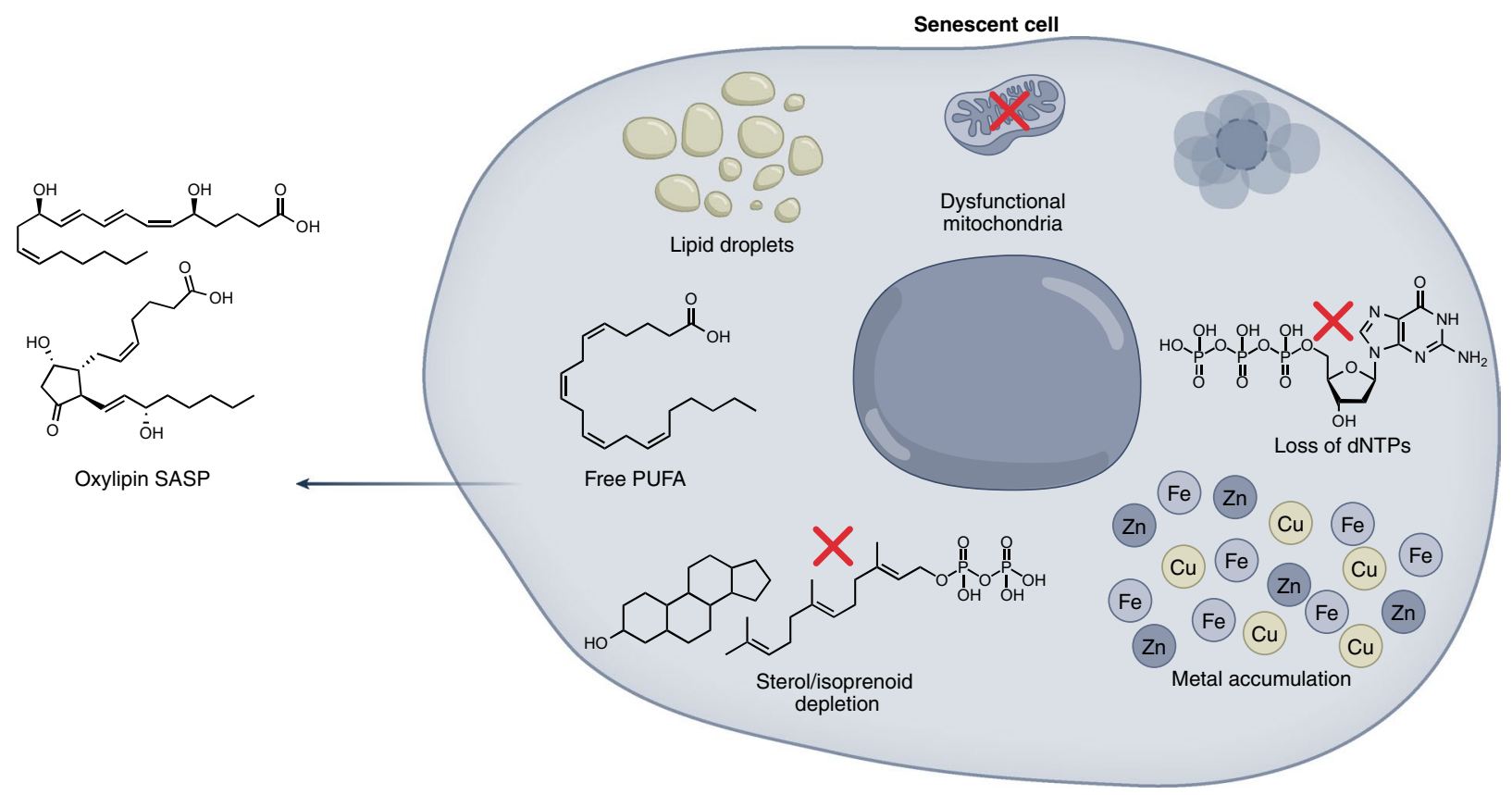

Fig. 3 | Altered metabolic states of senescent cells. Senescent cells have increased free cytosolic PUFAs, which in turn can be converted into oxylipins as part of a lipid-based SASP. Fatty acids also accumulate in lipid droplets during senescence. Sterols preferentially accumulate in the ER of some senescent cells in a p53-dependent manner, inhibiting sterol response element-binding protein 2 (SREBP2) activation and lowering sterol synthesis in the cell as a whole. Senescent cells often have increased mitochondrial mass or mtDNA, but this is coupled to altered membrane potential and ROS production. Lysosomes of senescent cells can become permeable, acidifying the cytosol and disrupting some forms of autophagy. This can lead to accumulation of transition metals inside senescent cells. Finally, senescent cells can have lower dNTP levels due to loss of ribonucleotide reductase 2. Note that senescence is a complex and varied phenomenon, and it is likely that individual metabolic alterations may be present in some, but not all, forms of senescence.

and senescence-associated dihomo-15d-PGJ2. These electrophilic prostaglandins have multiple activities inside cells. Primarily, they chage the structure of proteins by Michael addition to exposed cysteines ${ }^{133}$ - that is, the nucleophilic addition of a carbanion or other nucleophile to an $\alpha, \beta$-unsaturated carbonyl compound containing an electron withdrawing group.

The intracellular accumulation of dihomo-15d-PGJ2 in senescent cells promotes the activation of endogenous HRAS, thereby reinforcing senescence in a manner analogous to oncogene activation $^{124}$. Notably, this property of senescent cells allows the detection of senolysis because dihomo-15d-PGJ2 is only $~ 343 \mathrm{Da}$ in size and thus escapes the cytosol during apoptosis. Consequently, it can be detected in culture media or biological fluids upon senolysis $^{124}$. Together, these studies demonstrate that senescence and lipid metabolism are intricately linked (Fig. 4), but also highlight the importance of future work, as many areas of lipid metabolism are still underexplored in the context of senescence.

Lysosomes and autophagy. Autophagy, the process by which a cell breaks down its organelles and macromolecules, is dysregulated in senescent cells. Specific factors show selective microautophagic degradation in senescent cells, whereas macroautophagy appears to be less active in senescent cells ${ }^{134,135}$. Inhibition of autophagy by depletion of ATG7, ATG12 or LAMP2 induces senescence ${ }^{136}$, implying that loss of autophagic homeostasis during senescence may reinforce the senescence arrest and maintain the senescent state. However, activation of autophagy is associated with oncogene-induced senescence, and overexpression of ULK3 also promotes senescence ${ }^{137}$. The complex nature of the relationship between senescence and autophagy are the subject of recent reviews ${ }^{135,138}$.

Notably, many senescent cells have dysfunctional lysosomes. Indeed, SA-gal is a lysosomal $\beta$-galactosidase that is markedly upregulated in senescent cells ${ }^{139,140}$, and SA- $\beta$ gal may reflect lysosomal dysfunction. Recent findings show that lysosomal permeability increases in senescent cells. This increase results in the previously observed loss of lysosomal acidity ${ }^{141,142}$, but also elevates cytosolic acidification $^{143}$. To combat this increase, senescent cells upregulate glutaminolysis by increasing glutaminase 1 (GLS1) expression, which increases ammonia production and neutralizes the cytosolic acidity $^{143}$. Inhibition of GLS1 kills senescent cells both in culture and in vivo and ameliorates several indicators of age-related organ dysfunction $^{143}$. Therefore, lysosomal dysfunction, while potentially deleterious, is an exploitable weakness for targeted elimination of senescent cells.

Disruption of transition metal homeostasis. Transition metals-divalent cations that occupy a central block in the periodic table-play a key role in several cellular processes, but the role of these metals in senescence is only beginning to be understood. Surprisingly, senescent cells were found to accumulate transition metals at much higher levels than non-senescent or immortalized cells $^{144}$. These transition metals included iron, manganese and zinc. Later, other transition metals, such as copper ${ }^{145}$, were found to accumulate in senescent cells.

Iron. Iron levels are tightly controlled in most cells. Iron is required for oxygen transport, respiration and oxidative phosphorylation ${ }^{146}$. However, too much unbound or 'labile' iron can catalyze the production of ROS and peroxidized lipids, which can drive toxic cell processes, including a form of iron-dependent cell death known as ferroptosis ${ }^{147}$. Iron overload is antagonized by the protein ferritin, which binds and sequesters iron in an inactive form. Senescent cells import increased levels of iron by elevating the transferrin receptor, which then endocytoses iron-bound transferrin ${ }^{148}$. 


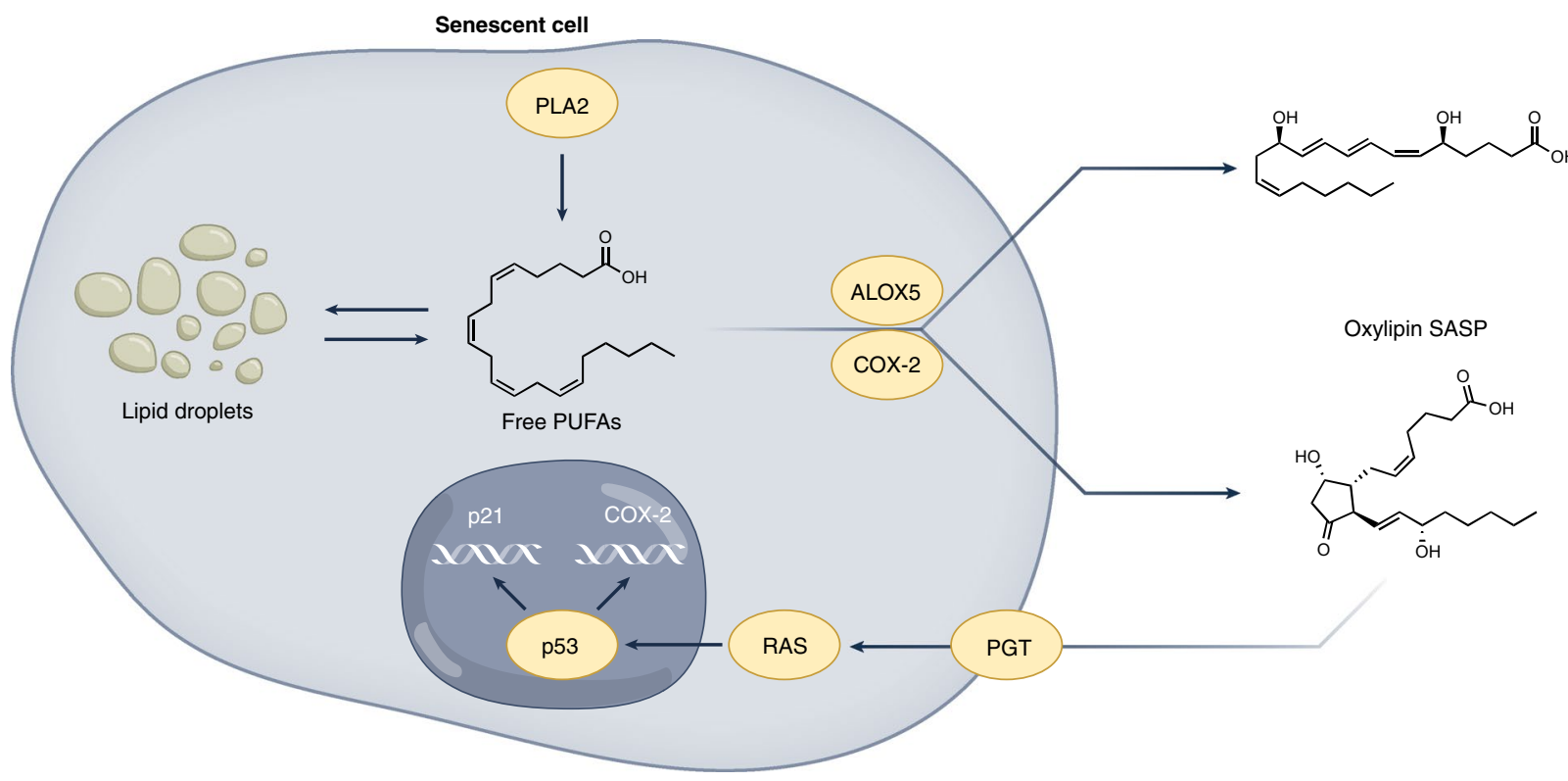

Fig. 4 | Lipid metabolism in senescent cells. Activation of phospholipase A2 (PLA2) frees PUFAs from the plasma membrane, which then accumulate in the form of triglycerides in lipid droplets but are also used as substrates for oxylipin synthases such as arachidonate 5-lipoxygenase (ALOX5) and cyclooxygenase 2 (COX-2), resulting in release of an oxylipin SASP. PGT imports prostaglandins into the cytosol, where cyclopentenone prostaglandins activate RAS, leading to p53 activation, leading to both cell cycle arrest p21 and elevation of COX-2, reinforcing oxylipin biosynthesis.

Ferritin levels increase in senescent cells, sequestering much of the imported iron in an inactive state ${ }^{148}$. Because iron is released from ferritin by targeted autophagy (ferritinophagy), and senescent cells display compromised autophagy and lysosomal dysfunction, senescent cells likely accumulate iron at least in part due to failure to degrade ferritin. Activation of autophagy by rapamycin partially restored cellular iron levels ${ }^{148}$. While iron-metabolizing protein levels suggest that labile iron may actually decrease during senescence $^{148}$, no reports of direct measurement of the labile iron pool in senescent cells exist to date. If the labile iron pool does indeed decrease during senescence, this finding might explain the observation that senescent cells are resistant to ferroptosis, as lack of labile iron would prevent iron-catalyzed lipid peroxidation. However, rapamycin had no effect on ferroptosis resistance in senescent cells, emphasizing the need for more research in this area.

Copper. Copper is an essential trace element required for the activity of multiple enzymes, including superoxide dismutase, neurotransmitter synthases, amine oxidases, ceruloplasmin and lysyl oxidase ${ }^{149}$. Excess copper can promote senescence in human fibroblasts ${ }^{150}$ and human glioblastoma cells by downregulating the polycomb protein Bmi-1 (ref. ${ }^{151}$ ), although both copper and oxygen levels were supraphysiological in these studies. Similar to iron, copper accumulates in senescent cells ${ }^{145}$, and, much like iron, this accumulation appears due to defective lysosomal autophagic degradation of copper chaperones. However, since copper-dependent antioxidant enzymes (for example, superoxide dismutase and glutathione reductase) are elevated during senescence ${ }^{145}$, and prevent senescence ${ }^{152}$, this elevation may be a compensatory mechanism to counteract the more oxidative environment observed in senescent cells. Much like iron, rapamycin partially abrogates copper accumulation in senescent cells ${ }^{145}$, suggesting regulation by autophagy, but the consequences of this elevation are unclear.

Zinc. Zinc is notable for its essential use by many organisms. One reason for this use is that, unlike iron and copper, zinc does not catalyze the formation of radicals from lipid peroxides ${ }^{153}$. Thus, zinc is less toxic and more redox inactive than iron and copper.
Nonetheless, zinc levels are inversely associated with lifespan in Caenorhabditis elegans ${ }^{154}$. Zinc levels also increase in senescent cells, and are generally higher than iron or copper levels ${ }^{144}$. Excess zinc promotes senescence in cultured vascular smooth muscle cells ${ }^{155}$, endothelial cells ${ }^{156}$ and fibroblasts ${ }^{157}$. Despite these studies showing an elevation of transition metals during senescence, no functional significance has yet been ascribed to the iron, copper or zinc accumulation in senescent cells. Since overload of these metals drives senescence, this accumulation may enforce a cell cycle arrest program that maintains senescent cells in an essentially irreversible growth-arrested state.

Senescence and dNTP synthesis. As senescent cells do not divide, their principal uses for deoxynucleoside triphosphates (dNTPs) are for DNA repair and mitochondria DNA synthesis. Ribose-linked ATP, GTP and CTP are converted to dNTPs by ribonucleotide reductase (RRM2). Unsurprisingly, RRM2 declines during senescence $^{158}$. However, when senescence is induced by oncogenic RAS, RRM2 depletion actually precedes the growth arrest and occurs during the hyperproliferative phase prior to cell cycle arrest ${ }^{158}$. Increased proliferation in the absence of dNTPs causes replication fork collapse, DNA double-strand breaks and cell cycle arrest. Addition of exogenous dNTPs allows continued proliferation in this model ${ }^{158}$. Eliminating p53 or p16 allows cells to bypass oncogenic RAS-induced senescence by increasing nucleotide synthesis via shunting metabolites through the pentose phosphate pathway $^{159,160}$. Notably, RAS activation antagonizes the hippo pathway transducers YAP/TAZ, and activation of this pathway restores RRM2 expression and allows escape from RAS-induced senescence $^{161}$. These findings suggest that senescent cells integrate external cues to control DNA metabolism.

\section{Interventions that reciprocally target senescence and metabolism}

As described above, senescence and metabolism and intricately linked. It would therefore follow that interventions that target metabolism might influence the accumulation of senescent cellsor properties of senescent cells, such as the SASP. Conversely, 
targeting senescent cells with, for example, senolytic drugs, should influence the development of metabolic disorders, as shown in Fig. 1 (right). In this section, we explore some of these links.

Senolytics as regulators of metabolism. Transgenic mice and drugs that allow the selective killing of senescent cells (senolysis/senolytic drugs) reveal that senescent cells promote many age-related conditions, thereby limiting both lifespan and health $\operatorname{span}^{48,49}$. Senolysis prevents several pathologies, including chronic kidney disease ${ }^{48,162}$, osteopenia $^{163}$ and osteoarthritis ${ }^{164}$. Senescent cells also promote tumorigenesis ${ }^{48,165}$, including both cancer relapse and several side effects of anti-cancer chemotherapy ${ }^{166,167}$. It is therefore not surprising that senescent cells also promote aspects of metabolic disease.

Senescent cells promote both diabetes and the degenerative complications of diabetes. Diabetes can be driven by pancreatic cell senescence, promoting insulitis and Type I diabetes ${ }^{168}$. Diabetes can also be driven by peripheral senescent cells in obese mice, which drive insulin resistance and Type 2 diabetes ${ }^{169}$. Surprisingly, increasing the presence of senescent cells by transgenically overexpressing p16 ${ }^{I N K 4 a}$ in pancreatic $\beta$-cells of mice actually increases insulin secretion $^{170}$, so the development of senescence may reflect metabolic compensation for high blood glucose levels. Similarly, insulin resistance can drive hyperinsulinemia and promote $\beta$-cell senescence ${ }^{171}$, implicating peripheral senescent cells as feedback drivers of senescence in the pancreas. Furthermore, complications of diabetes, including diabetic kidney disease ${ }^{172,173}$ and diabetic retinopathy ${ }^{174}$, potentially result from senescent cells driven by increased blood glucose levels, as described above. In each case, senolytic therapies improve health outcomes in terms of either blood glucose levels ${ }^{168,169,171}$ or development of diabetes-driven complications ${ }^{172,174}$. Together, these studies implicate senescent cells as a major nexus for interventions into diabetes and its associated disorders.

Dietary interventions and senescence. Multiple dietary interventions can limit the accumulation of senescent cells with age. Calorie restriction (CR) was one of the first lifespan-extending interventions to be identified ${ }^{175}$. Thus, when it was found that senescent cells can limit lifespan ${ }^{48}$, it seemed that links between CR and senescence were likely. CR lowers markers of senescence in the colons of mice and humans ${ }^{176}$ and in inguinal white adipose tissue ${ }^{177}$. CR prevents the development of senescence in the kidney of aged animals, whereas high-calorie diets increase senescence ${ }^{178}$.

Other dietary interventions can similarly extend health span and either or both median and maximum lifespan. These interventions include methionine restriction ${ }^{179}$ and ketogenic diets ${ }^{180,181}$. Methionine restriction lowers markers of senescence and the $\mathrm{SASP}^{182}$, while injection of mice with $\beta$-hydroxybutyrate, a major ketone body produced by ketogenic diets, lowers markers of senescence in vascular smooth muscle and endothelial cells ${ }^{183}$. These results indicate that dietary interventions that create a favorable metabolic state that can limit the accumulation of senescent cells.

Metabolic interventions that influence senescent cells. As described above, hyperglycemia induces senescence. It therefore reasons that diabetes could be pro-senescence, and interventions that lower blood sugar should antagonize the formation of senescent cells. Indeed, kidney proximal tubule cells become senescent within 4 weeks of induction of diabetes in mice, and this rise in senescent cells can be prevented by lowering glucose levels with insulin or inhibiting glucose transport into the cells by inhibiting the activity of the sodium-glucose co-transporter SGLT2 ${ }^{102}$. Similarly, acarbose-which lowers blood sugar by antagonizing intestinal carbohydrate absorption-extends lifespan in mice ${ }^{184}$ and prevents diet-induced atherosclerosis-associated senescence in rabbits ${ }^{185}$.

Another antidiabetic medication, metformin, extends both lifespan and health span in mice ${ }^{186}$, and is a drug being studied for the first clinical trial to monitor general aspects of aging (under review; see https://www.afar.org/tame-trial). Metformin antagonizes the development of senescence in response to ceramide in immortal mouse myoblast cells ${ }^{187}$ and extends the replicative lifespan of human fibroblasts and mesenchymal stem cells ${ }^{188}$. Additionally, metformin lowers several SASP factor levels, including many proinflammatory cytokines, by interfering with NF-kB activation ${ }^{189}$. Furthermore, metformin protects against the development of senescence in murine models of intervertebral disc degeneration ${ }^{190}$ and chronic kidney disease ${ }^{191}$. Metformin therefore interferes with senescence at several levels and is perhaps the best-characterized metabolic intervention that antagonizes senescence and the SASP.

Atherosclerosis, statins and senescence. Statins, such as simvastatin, are commonly used among older adults as preventives for elevated cholesterol levels and associated atherosclerosis-related complications, such as stroke and heart attack. They prevent cholesterol synthesis by inhibiting $\beta$-hydroxy $\beta$-methylglutaryl-coenzyme A (HMG-CoA) reductase, the rate-limiting enzyme in the mevalonate pathway that leads to cholesterol biosythesis ${ }^{192}$. Statins prevent the senescence growth arrest, with lowered markers of senescence, in endothelial progenitor cells ${ }^{193}$, which is dependent on lowered levels of farnesylpyrophosphate and geranylgeranylpyrophosphate $^{193}$. Statins also inhibit many aspects of the proinflammatory SASP of senescent fibroblasts, but, as with endothelial progenitor senescence, this effect is independent of the cholesterol-lowering properties of the drug ${ }^{194}$. Rather, HMG-CoA reductase inhibition lowers farnesyl-CoA and geranylgeranyl-CoA levels, which in turn are required for membrane localization and activation of rho-type GTPases, including RAS and RAC, which are required for the SASP. Since p16-positive (presumably senescent) foamy intimal cells promote deleterious features of atherosclerosis ${ }^{195}$, it is possible that statins may have anti-atherogenic properties beyond regulating cholesterol. However, statins can also act as radiosensitizersincreasing cancer cell senescence in response to irradiation ${ }^{196}$. So, the effects of statins are likely to be context-specific. Surprisingly, p53 activation during senescence limits both the mevalonate and cholesterol synthesis pathways ${ }^{197}$, and therefore may be a mechanism by which $\mathrm{p} 53$ activation limits aspects of the SASP ${ }^{15,66}$. The relationship between senescence, cholesterol and atherosclerosis is therefore likely to be more complex than previously thought.

Exercise as an intervention for senescence. While numerous studies highlight the health benefits of exercise and its improvement of health span with age, few studies to date have demonstrated lifespan extension due to exercise in mice ${ }^{198,199}$; just one late-life exercise study showed lifespan extension ${ }^{200}$. There is evidence that exercise can prevent senescence and segments of the proinflammatory SASP in the sera and hearts of aged mice ${ }^{199}$. More notably, exercise offsets diet-induced senescence and the SASP in mouse adipose and liver tissues $^{201}$, suggesting that the most beneficial aspects of exercise may not pertain to already healthy animals, but rather those likely to develop metabolic disease. In humans, exercise and activity are associated with reduced markers of endothelial and leukocyte cell senescence ${ }^{202,203}$. These data suggest that exercise can offset deleterious aspects of senescence brought about by poor diet and sedentary lifestyles.

\section{Conclusions}

As noted throughout this article, the interplay between senescent cells and systemic metabolism is dynamic. Senescent cells can disrupt metabolic homeostasis in multiple tissues, and these changes in turn can promote senescence, creating a feedback loop that can drive pathology. This loop occurs in the context of $\mathrm{NAD}^{+}$, where the SASP promotes CD38-dependent $\mathrm{NAD}^{+}$breakdown by immune cells ${ }^{95,96}$, while lowered $\mathrm{NAD}^{+}$levels in turn can promote senescence $^{39}$ (Fig. 2). Similarly, peripheral senescence can result in 


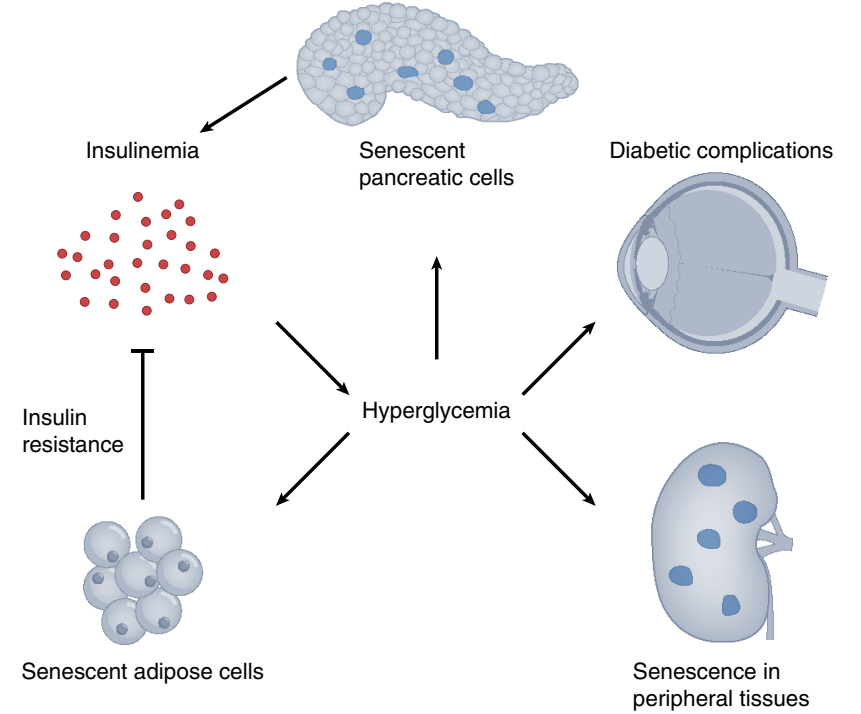

Fig. 5 | Multiple roles for senescence in diabetes and its complications. Senescent adipose cells can result in insulin resistance, which results in hyperglycemia, which can in turn promote additional adipose tissue senescence. Hyperglycemia also forces pancreatic $\beta$-cells to over-produce insulin. This stress results in $\beta$-cell senescence, leading to insulinemia, resulting in further hyperglycemia. Hyperglycemia also results in senescence in peripheral tissues such as the retina and the kidney, fueling diabetic complications.

insulin resistance ${ }^{169}$, while insulin resistance in turn drives stress and senescence in pancreatic $\beta$-cells ${ }^{171}$. This degenerative cycle leads to elevated blood glucose and senescence in the eye and kidney, ultimately driving diabetic complications ${ }^{172,174}$ (Fig. 5). These feedback loops require interventions that break the cycle in order to be efficacious.

There are three types of interventions that target senescent cells and their degenerative pathologies. First, we can slow the formation of senescent cells, as observed during dietary restriction and similar interventions. Second, we can allow senescent cells to accumulate, but prevent them from causing harm, as observed during metformin-mediated SASP suppression or after CD38 inhibition. Finally, we can use senolysis to remove senescent cells. Unlike the first two interventions, senolysis can be used intermittently-allowing for a 'hit and run' approach that might be easier to implement in humans-whereas dietary and suppressive drug regimens require regular adherence to maintain benefits.

The metabolic changes observed in senescent cells also imply that additional disorders might be driven by senescent cells. For example, it was recently shown that senescent cells occur in the chorioamnionic membrane prior to birth in mammals, and that these cells send signals to promote parturition ${ }^{28}$. Given that parturition is promoted by prostaglandins E2 (PGE2) and F2 $\alpha$ (PGF2 $\alpha$ ), it may be that senescence-associated prostaglandins are part of normal (and potentially premature) labor in humans. Prostaglandins also have additional roles, such as the essential role of PGD2 in sleep initiation ${ }^{204,205}$. Given that many people experience increased sleep disruption with age ${ }^{206}$, it is tempting to speculate that senescent cells may produce additional PGD2 that drives age-related sleep disruption.

Finally, age is a major outcome of human nutrition, while nutritional interventions can prevent many age-associated diseases ${ }^{207}$. Much is known about the role of macronutrient stress in longevity and senescence, but less is known about how malnutrition of key micronutrients controls the development of senescence. For example, choline deficiency is common in the elderly in the United
States $^{207}$, and is a driver of hepatic steatosis-a condition also promoted during age by senescent cells ${ }^{208}$. The relationship between nutrition and senescence is therefore fertile ground for future exploration and will undoubtedly be the subject of multiple studies moving forward.

Clearly, many aspects of the interplay between metabolism and cellular senescence need additional research. Encouragingly, however, emerging tools of modern biology, including single cell and systems biology and artificial intelligence, are making it easier to obtain deep insights into this complex interplay, which will undoubtedly lead to more efficacious interventions into age-related metabolic pathologies in the future.

Received: 9 June 2021; Accepted: 13 September 2021; Published online: 18 October 2021

\section{References}

1. Hayflick, L. The limited in vitro lifetime of human diploid cell strains. Exp. Cell. Res. 37, 614-636 (1965)

2. Hayflick, L. \& Moorhead, P. S. The serial cultivation of human diploid cell strains. Exp. Cell. Res. 25, 585-621 (1961).

3. Carrel, A. On the permanent life of tissues outside of the organism. J. Exp. Med. 15, 516-528 (1912).

4. Sager, R., Tanaka, K., Lau, C. C., Ebina, Y. \& Anisowicz, A. Resistance of human cells to tumorigenesis induced by cloned transforming genes. Proc. Natl Acad. Sci. USA 80, 7601-7605 (1984).

5. Sager, R. Senescence as a mode of tumor suppression. Environ. Health Persp. 93, 59-62 (1991).

6. Campisi, J. Aging, cellular senescence, and cancer. Annu. Rev. Physiol. 75, 685-705 (2013).

7. Collado, M., Blasco, M. A. \& Serrano, M. Cellular senescence in cancer and aging. Cell 130, 223-233 (2007).

8. de Keizer, P. L., Laberge, R. M. \& Campisi, J. p53: Pro-aging or pro-longevity? Aging 2, 377-379 (2010).

9. Chicas, A. et al. Dissecting the unique role of the retinoblastoma tumor suppressor during cellular senescence. Cancer Cell 17, 376-387 (2010).

10. Chang, J. et al. Clearance of senescent cells by ABT263 rejuvenates aged hematopoietic stem cells in mice. Nat. Med. 22, 78-83 (2016).

11. Yosef, R. et al. Directed elimination of senescent cells by inhibition of BCL-W and BCL-XL. Nat. Commun. 7, 11190 (2016).

12. Zhu, Y. et al. Identification of a novel senolytic agent, navitoclax, targeting the Bcl-2 family of anti-apoptotic factors. Aging Cell 15, 428-435 (2016).

13. Kim, D. E. et al. Deficiency in the DNA repair protein ERCC1 triggers a link between senescence and apoptosis in human fibroblasts and mouse skin. Aging Cell 19, e13072 (2020).

14. Basisty, N. et al. A proteomic atlas of senescence-associated secretomes for aging biomarker development. PLoS Biol. 18, e3000599 (2020).

15. Coppe, J. P. et al. Senescence-associated secretory phenotypes reveal cell non-automous functions of oncogenic RAS and the p53 tumor suppressor. PLoS Biol. 6, 2853-2868 (2008).

16. Cevenini, E., Monti, D. \& Franceschi, C. Inflamm-ageing. Curr. Opin. Clin. Nutr. Metab. Care 16, 14-20 (2013).

17. Franceschi, C. \& Campisi, J. Chronic inflammation (inflammaging) and its potential contribution to age-associated diseases. J. Gerontol. 69, 54-59 (2014).

18. Furman, D. et al. Chronic inflammation in the etiology of disease across the life span. Nat. Med. 25, 1822-1832 (2019).

19. Hernandez-Segura, A. et al. Unmasking transcriptional heterogeneity in senescent cells. Curr. Biol. 27, 2652-2660 (2017).

20. Alimonti, A. et al. A novel type of cellular senescence that can be enhanced in mouse models and human tumor xenografts to suppress prostate tumorigenesis. J. Clin. Invest. 120, 681-693 (2010).

21. Dimri, G. P. et al. A novel biomarker identifies senescent human cells in culture and in aging skin in vivo. Proc. Natl Acad. Sci. USA 92, 9363-9367 (1995).

22. Freund, A., Laberge, R. M., Demaria, M. \& Campisi, J. Lamin B1 loss is senescence-associated biomarker. Molec. Biol. Cell 23, 2066-2075 (2012).

23. Davalos, A. R. et al. p53-dependent release of Alarmin HMGB1 is a central mediator of senescent phenotypes. J. Cell Biol. 201, 613-629 (2013).

24. Gorgoulis, V. et al. Cellular Senescence: Defining a Path Forward. Cell 179, 813-827 (2019).

25. Williams, G. C. Pleiotropy, natural selection, and the evolution of senescence. Evolution 11, 398-411 (1957).

26. Muñoz-Espín, D. et al. Programmed cell senescence during mammalian embryonic development. Cell 155, 1104-1118 (2013).

27. Storer, M. et al. Senescence is a developmental mechanism that contributes to embryonic growth and patterning. Cell 155, 1119-1130 (2013). 
28. Menon, R., Richardson, L. S. \& Lappas, M. Fetal membrane architecture, aging and inflammation in pregnancy and parturition. Placenta 79, 40-45 (2019).

29. Menon, R. et al. Placental membrane aging and HMGB1 signaling associated with human parturition. Aging 8, 216-230 (2016).

30. Chu, X., Wen, J. \& Raju, R. P. Rapid senescence-like response after acute injury. Aging Cell 19, el3201 (2020).

31. Demaria, M. et al. An essential role for senescent cells in optimal wound healing through secretion of PDGF-AA. Dev. Cell 31, 722-733 (2014).

32. Kortlever, R. M. \& Bernards, R. Senescence, wound healing and cancer: the PAI-1 connection. Cell Cycle 5, 2697-2703 (2006).

33. Ritschka, B. et al. The senescence-associated secretory phenotype induces cellular plasticity and tissue regeneration. Genes Dev. 31, 172-183 (2017).

34. Sarig, R. et al. Transient p53-mediated regenerative senescence in the injured heart. Circulation 139, 2491-2494 (2019).

35. Adams, P. D. Healing and hurting: molecular mechanisms, functions and pathologies of cellular senescence. Molec. Cell 36, 2-14 (2009).

36. Baker, D. J. \& Sedivy, J. M. Probing the depths of cellular senescence. J. Cell Biol. 202, 11-13 (2013)

37. Childs, B. G. et al. Senescent cells: an emerging target for diseases of ageing. Nat. Rev. Drug Disc. 16, 718-735 (2017).

38. Tominaga, $\mathrm{K}$. The emerging role of senescent cells in tissue homeostasis and pathophysiology. Pathobiol. Aging Age Rel. Dis. 5, 27743 (2015)

39. Wiley, C. D. \& Campisi, J. From Ancient Pathways to Aging Cells-Connecting Metabolism and Cellular Senescence. Cell Metab. 23, 1013-1021 (2016).

40. Trifunovic, A. et al. Premature ageing in mice expressing defective mitochondrial DNA polymerase. Nature 429, 417-423 (2004).

41. Kujoth, G. C. et al. Mitochondrial DNA mutations, oxidative stress, and apoptosis in mammalian aging. Science 309, 481-484 (2005).

42. Schriner, S. E. et al. Extension of murine life span by overexpression of catalase targeted to mitochondria. Science 308, 1909-1911 (2005).

43. Wiley, C. D. et al. Mitochondrial Dysfunction Induces Senescence with a Distinct Secretory Phenotype. Cell Metab. 23, 303-314 (2016).

44. Miwa, S. et al. Low abundance of the matrix arm of complex I in mitochondria predicts longevity in mice. Nat. Commun. 5, 3837 (2014).

45. Velarde, M. C., Flynn, J. M., Day, N. U., Melov, S. \& Campisi, J. Mitochondrial oxidative stress caused by Sod2 deficiency promotes cellular senescence and aging phenotypes in the skin. Aging 4, 3-12 (2012).

46. Correia-Melo, C. et al. Mitochondria are required for pro-ageing features of the senescent phenotype. EMBO J. 35, 724-742 (2016).

47. Vizioli, M. G. et al. Mitochondria-to-nucleus retrograde signaling drives formation of cytoplasmic chromatin and inflammation in senescence. Genes Dev. 34, 428-445 (2020).

48. Baker, D. J. et al. Naturally occurring p16(Ink4a)-positive cells shorten healthy lifespan. Nature 530, 184-189 (2016).

49. Xu, M. et al. Senolytics improve physical function and increase lifespan in old age. Nat. Med. 24, 1246-1256 (2018).

50. Perez, V. I. et al. The overexpression of major antioxidant enzymes does not extend the lifespan of mice. Aging Cell 8, 73-75 (2009).

51. Diebold, L. \& Chandel, N. S. Mitochondrial ROS regulation of proliferating cells. Free Radic. Biol. Med. 100, 86-93 (2016).

52. West, A. P., Shadel, G. S. \& Ghosh, S. Mitochondria in innate immune responses. Nat. Rev. Immunol. 11, 389-402 (2011).

53. Morimoto, $\mathrm{H}$. et al. ROS are required for mouse spermatogonial stem cell self-renewal. Cell Stem Cell 12, 774-786 (2013).

54. Juntilla, M. M. et al. AKT1 and AKT2 maintain hematopoietic stem cell function by regulating reactive oxygen species. Blood 115, 4030-4038 (2010).

55. Tormos, K. V. et al. Mitochondrial complex III ROS regulate adipocyte differentiation. Cell Metab. 14, 537-544 (2011).

56. Maryanovich, M. \& Gross, A. A ROS rheostat for cell fate regulation. Trends Cell Biol. 23, 129-134 (2013).

57. Ziegler, D. V. et al. Calcium channel ITPR2 and mitochondria-ER contacts promote cellular senescence and aging. Nat. Commun. 12, 720 (2021).

58. Vasileiou, P. V. S. et al. Mitochondrial Homeostasis and Cellular Senescence. Cells 8, https://doi.org/10.3390/cells8070686 (2019).

59. Chapman, J., Fielder, E. \& Passos, J. F. Mitochondrial dysfunction and cell senescence: deciphering a complex relationship. FEBS Lett. $\mathbf{5 9 3}$, 1566-1579 (2019).

60. Korolchuk, V. I., Miwa, S., Carroll, B. \& von Zglinicki, T. Mitochondria in Cell Senescence: Is Mitophagy the Weakest Link? EBioMedicine 21, 7-13 (2017).

61. Ziegler, D. V., Wiley, C. D. \& Velarde, M. C. Mitochondrial effectors of cellular senescence: beyond the free radical theory of aging. Aging Cell $\mathbf{1 4}$ 1-7 (2015).

62. Parrinello, S. et al. Oxygen sensitivity severely limits the replicative lifespan of murine fibroblasts. Nat. Cell Biol. 5, 741-747 (2003).

63. Nakazawa, M. S., Keith, B. \& Simon, M. C. Oxygen availability and metabolic adaptations. Nat. Rev. Cancer 16, 663-673 (2016).
64. Casciaro, F. et al. Prolonged hypoxia delays aging and preserves functionality of human amniotic fluid stem cells. Mech. Ageing Dev. 191, 111328 (2020).

65. Leontieva, O. V. et al. Hypoxia suppresses conversion from proliferative arrest to cellular senescence. Proc. Natl Acad. Sci. USA 109 13314-13318 (2012).

66. Wiley, C. D. et al. Small-molecule MDM2 antagonists attenuate the senescence-associated secretory phenotype. Sci. Rep. 8, 2410 (2018)

67. Coppe, J. P. et al. A human-like senescence-associated secretory phenotype is conserved in mouse cells dependent on physiological oxygen. PLoS ONE 5, e9188 (2010).

68. van Vliet, T. et al. Physiological hypoxia restrains the senescence-associated secretory phenotype via AMPK-mediated mTOR suppression. Mol. Cell 81, 2041-2052 e2046 (2021)

69. Ast, T. \& Mootha, V. K. Oxygen and mammalian cell culture: are we repeating the experiment of Dr. Ox? Nat. Metab. 1, 858-860 (2019).

70. Baik, A. H. \& Jain, I. H. Turning the Oxygen Dial: Balancing the Highs and Lows. Trends Cell Biol. 30, 516-536 (2020).

71. van Vliet, T., Casciaro, F. \& Demaria, M. To breathe or not to breathe: Understanding how oxygen sensing contributes to age-related phenotypes. Ageing Res. Rev. 67, 101267 (2021).

72. Parikh, P. et al. Hyperoxia-induced Cellular Senescence in Fetal Airway Smooth Muscle Cells. Am. J. Respir. Cell Mol. Biol. 61, 51-60 (2019).

73. Daher, A. et al. Clinical course of COVID-19 patients needing supplemental oxygen outside the intensive care unit. Sci. Rep. 11, 2256 (2021).

74. You, K. et al. Moderate hyperoxia induces senescence in developing human lung fibroblasts. Am. J. Physiol. Lung Cell. Mol. Physiol. 317, L525-L536 (2019).

75. Hachmo, Y. et al. Hyperbaric oxygen therapy increases telomere length and decreases immunosenescence in isolated blood cells: a prospective trial. Aging 12, 22445-22456 (2020).

76. Dookun, E. et al. Clearance of senescent cells during cardiac ischemia-reperfusion injury improves recovery. Aging Cell 19, e13249 (2020)

77. Fleury, H. et al. Exploiting interconnected synthetic lethal interactions between PARP inhibition and cancer cell reversible senescence. Nat. Commun. 10, 2556 (2019)

78. Ohanna, M. et al. Senescent cells develop a PARP-1 and nuclear factor\{kappa\}B-associated secretome (PNAS). Genes Dev. 25, 1245-1261 (2011).

79. Nassour, J. et al. Defective DNA single-strand break repair is responsible for senescence and neoplastic escape of epithelial cells. Nat. Commun. 7, 10399 (2016).

80. Nacarelli, T. et al. $\mathrm{NAD}(+)$ metabolism governs the proinflammatory senescence-associated secretome. Nat. Cell Biol. 21, 397-407 (2019).

81. Gomes, A. P. et al. Declining $\mathrm{NAD}(+)$ induces a pseudohypoxic state disrupting nuclear-mitochondrial communication during aging. Cell $\mathbf{1 5 5}$, 1624-1638 (2013)

82. Yoshino, J., Mills, K. F., Yoon, M. J. \& Imai, S. Nicotinamide mononucleotide, a key $\mathrm{NAD}(+)$ intermediate, treats the pathophysiology of diet- and age-induced diabetes in mice. Cell Metab. 14, 528-536 (2011).

83. Zhang, $\mathrm{H}$. et al. $\mathrm{NAD}(+)$ repletion improves mitochondrial and stem cell function and enhances life span in mice. Science 352, 1436-1443 (2016).

84. Baker, D. J. et al. Increased expression of BubR1 protects against aneuploidy and cancer and extends healthy lifespan. Nat. Cell Biol. 15, 96-102 (2012)

85. Andriani, G. A. et al. Whole chromosome instability induces senescence and promotes the SASP. Sci. Rep. 6, 35218 (2016).

86. North, B. J. et al. SIRT2 induces the checkpoint kinase BubR1 to increase lifespan. EMBO J. 33, 1438-1453 (2014)

87. Simon, M. et al. LINE1 Derepression in Aged Wild-Type and SIRT6-Deficient Mice Drives Inflammation. Cell Metab. 29, 871-885 e875 (2019).

88. De Cecco, M. et al. L1 drives IFN in senescent cells and promotes age-associated inflammation. Nature 566, 73-78 (2019).

89. Mao, Z. et al. Sirtuin 6 (SIRT6) rescues the decline of homologous recombination repair during replicative senescence. Proc. Natl Acad. Sci. USA 109, 11800-11805 (2012).

90. $\mathrm{Xu}, \mathrm{C}$. et al. SIRT1 is downregulated by autophagy in senescence and ageing. Nat. Cell Biol. 22, 1170-1179 (2020).

91. Hayakawa, T. et al. SIRT1 suppresses the senescence-associated secretory phenotype through epigenetic gene regulation. PLOS ONE 10, e0116480 (2015)

92. Ota, H. et al. Sirtl inhibitor, Sirtinol, induces senescence-like growth arrest with attenuated Ras-MAPK signaling in human cancer cells. Oncogene 25, 176-185 (2006)

93. Bonkowski, M. S. \& Sinclair, D. A. Slowing ageing by design: the rise of $\mathrm{NAD}(+)$ and sirtuin-activating compounds. Nat. Rev. Mol. Cell Biol. 17 679-690 (2016).

94. Covarrubias, A. J., Perrone, R., Grozio, A. \& Verdin, E. NAD $(+)$ metabolism and its roles in cellular processes during ageing. Nat. Rev. Mol. Cell Biol. 22, 119-141 (2021) 
95. Covarrubias, A. J. et al. Senescent cells promote tissue NAD $(+)$ decline during ageing via the activation of $\mathrm{CD} 38(+)$ macrophages. Nat. Metab. 2, 1265-1283 (2020).

96. Chini, C. C. S. et al. CD38 ecto-enzyme in immune cells is induced during aging and regulates $\mathrm{NAD}(+)$ and NMN levels. Nat. Metab. 2, 1284-1304 (2020).

97. Camacho-Pereira, J. et al. CD38 dictates age-related NAD decline and mitochondrial dysfunction through an SIRT3-dependent mechanism. Cell Metab. 23, 1127-1139 (2016).

98. Lee, S. M. et al. Cytosolic malate dehydrogenase regulates senescence in human fibroblasts. Biogerontology 13, 525-536 (2012).

99. Jiang, P., Du, W., Mancuso, A., Wellen, K. E. \& Yang, X. Reciprocal regulation of p53 and malic enzymes modulates metabolism and senescence. Nature 493, 689-693 (2013).

100. Blazer, S. et al. High glucose-induced replicative senescence: point of no return and effect of telomerase. Biochem. Biophys. Res. Commun. 296, 93-101 (2002)

101. Patel, C. et al. Arginase as a mediator of diabetic retinopathy. Front Immunol. 4, 173 (2013)

102. Kitada, K. et al. Hyperglycemia causes cellular senescence via a SGLT2- and p21-dependent pathway in proximal tubules in the early stage of diabetic nephropathy. J. Diabetes Complications 28, 604-611 (2014).

103. Mortuza, R., Chen, S., Feng, B., Sen, S. \& Chakrabarti, S. High glucose induced alteration of SIRTs in endothelial cells causes rapid aging in a p300 and FOXO regulated pathway. PLoS ONE 8, e54514 (2013).

104. Zhang, B. et al. SIRT3 overexpression antagonizes high glucose accelerated cellular senescence in human diploid fibroblasts via the SIRT3-FOXO1 signaling pathway. Age 35, 2237-2253 (2013).

105. Shosha, E. et al. Mechanisms of diabetes-induced endothelial cell senescence: role of Arginase 1. Int. J. Mol. Sci. https://doi.org/10.3390/ ijms19041215 (2018)

106. Hayashi, T. et al. Endothelial cellular senescence is inhibited by nitric oxide: implications in atherosclerosis associated with menopause and diabetes. Proc. Natl Acad. Sci. USA 103, 17018-17023 (2006).

107. Jin, J. \& Zhang, T. Effects of glucose restriction on replicative senescence of human diploid fibroblasts IMR-90. Cell. Physiol. Biochem. 31, 718-727 (2013).

108. Sohn, J. J. et al. Macrophages, nitric oxide and microRNAs are associated with DNA damage response pathway and senescence in inflammatory bowel disease. PLOS ONE 7, e44156 (2012).

109. Zhang, Y. et al. The pivotal role of protein acetylation in linking glucose and fatty acid metabolism to beta-cell function. Cell Death Dis. 10, 66 (2019).

110. Meyer, J. G. et al. Temporal dynamics of liver mitochondrial protein acetylation and succinylation and metabolites due to high fat diet and/or excess glucose or fructose. PLoS ONE 13, e0208973 (2018).

111. Wu, J., Jin, Z., Zheng, H. \& Yan, L. J. Sources and implications of NADH/ $\mathrm{NAD}(+)$ redox imbalance in diabetes and its complications. Diabetes Metab. Syndr. Obes. 9, 145-153 (2016).

112. Liu, J. et al. Receptor for advanced glycation end-products promotes premature senescence of proximal tubular epithelial cells via activation of endoplasmic reticulum stress-dependent p21 signaling. Cell Signal 26, 110-121 (2014).

113. Liu, J. et al. Impact of ER stress-regulated ATF4/p16 signaling on the premature senescence of renal tubular epithelial cells in diabetic nephropathy. Am. J. Physiol. Cell Physiol. 308, C621-C630 (2015).

114. Chen, C. Y., Abell, A. M., Moon, Y. S. \& Kim, K. H. An advanced glycation end product (AGE)-receptor for AGEs (RAGE) axis restores adipogenic potential of senescent preadipocytes through modulation of $\mathrm{p} 53$ protein function. J. Biol. Chem. 287, 44498-44507 (2012).

115. Venable, M. E., Lee, J. Y., Smyth, M. J., Bielawska, A. \& Obeid, L. M. Role of ceramide in cellular senescence. J. Biol. Chem. 270, 30701-30708 (1995).

116. Venable, M. E. \& Yin, X. Ceramide induces endothelial cell senescence. Cell Biochem. Funct. 27, 547-551 (2009).

117. Lee, J. Y., Bielawska, A. E. \& Obeid, L. M. Regulation of cyclin-dependent kinase 2 activity by ceramide. Exp. Cell. Res. 261, 303-311 (2000).

118. Chao, R., Khan, W. \& Hannun, Y. A. Retinoblastoma protein dephosphorylation induced by D-erythro-sphingosine. J. Biol. Chem. 267, 23459-23462 (1992).

119. Trayssac, M., Hannun, Y. A. \& Obeid, L. M. Role of sphingolipids in senescence: implication in aging and age-related diseases. J. Clin. Invest. 128, 2702-2712 (2018)

120. Quijano, C. et al. Oncogene-induced senescence results in marked metabolic and bioenergetic alterations. Cell Cycle 11, 1383-1392 (2012).

121. Fafian-Labora, J. et al. FASN activity is important for the initial stages of the induction of senescence. Cell Death Dis. 10, 318 (2019).

122. Flor, A. C., Wolfgeher, D., Wu, D. \& Kron, S. J. A signature of enhanced lipid metabolism, lipid peroxidation and aldehyde stress in therapy-induced senescence. Cell Death Discov. 3, 17075 (2017).
123. Maeda, M., Scaglia, N. \& Igal, R. A. Regulation of fatty acid synthesis and Delta9-desaturation in senescence of human fibroblasts. Life Sci. $\mathbf{8 4}$ 119-124 (2009).

124. Wiley, C. D. et al. Oxylipin biosynthesis reinforces cellular senescence and allows detection of senolysis. Cell Metab. https://doi.org/10.1016/j.cmet. 2021.03.008 (2021).

125. Wiley, C. D. et al. Secretion of leukotrienes by senescent lung fibroblasts promotes pulmonary fibrosis. JCI Insight https://doi.org/10.1172/jci.insight. 130056 (2019).

126. Lizardo, D. Y., Lin, Y. L., Gokcumen, O. \& Atilla-Gokcumen, G. E. Regulation of lipids is central to replicative senescence. Mol. Biosyst. 13, 498-509 (2017).

127. Ogrodnik, M. et al. Obesity-induced cellular senescence drives anxiety and impairs neurogenesis. Cell Metab. 29, 1233 (2019).

128. Kim, W. et al. Polyunsaturated fatty acid desaturation is a mechanism for glycolytic NAD(+) recycling. Cell Metab. 29, 856-870 (2019).

129. Catalano, A., Rodilossi, S., Caprari, P., Coppola, V. \& Procopio, A. 5-Lipoxygenase regulates senescence-like growth arrest by promoting ROS-dependent p53 activation. EMBO J. 24, 170-179 (2005).

130. Martien, S. et al. Cellular senescence involves an intracrine prostaglandin E2 pathway in human fibroblasts. Biochim. Biophys. Acta 1831, 1217-1227 (2013).

131. Schafer, M. J. et al. Cellular senescence mediates fibrotic pulmonary disease. Nat. Commun. 8, 14532 (2017)

132. Han, J. H. et al. Selective COX-2 inhibitor, NS-398, inhibits the replicative senescence of cultured dermal fibroblasts. Mech. Ageing Dev. 125, 359-366 (2004).

133. Shibata, T. et al. 15-deoxy-delta 12,14-prostaglandin J2. A prostaglandin D2 metabolite generated during inflammatory processes. J. Biol. Chem. 277, 10459-10466 (2002).

134. Kang, C. \& Elledge, S. J. How autophagy both activates and inhibits cellular senescence. Autophagy 12, 898-899 (2016).

135. Kwon, Y., Kim, J. W., Jeoung, J. A., Kim, M. S. \& Kang, C. Autophagy is pro-senescence when seen in close-up, but anti-senescence in long-shot. Mol. Cells 40, 607-612 (2017).

136. Kang, H. T., Lee, K. B., Kim, S. Y., Choi, H. R. \& Park, S. C. Autophagy impairment induces premature senescence in primary human fibroblasts. PLoS ONE 6, e23367 (2011).

137. Young, A. R. et al. Autophagy mediates the mitotic senescence transition. Genes Dev. 23, 798-803 (2009).

138. Rajendran, P. et al. Autophagy and senescence: a new insight in selected human diseases. J. Cell. Physiol. 234, 21485-21492 (2019).

139. Kurz, D. J., Decary, S., Hong, Y. \& Erusalimsky, J. D. Senescence-associated (beta)-galactosidase reflects an increase in lysosomal mass during replicative ageing of human endothelial cells. J. Cell Sci. 113, 3613-3622 (2000).

140. Lee, B. Y. et al. Senescence-associated beta-galactosidase is lysosomal beta-galactosidase. Aging Cell 5, 187-195 (2006).

141. Han, L. et al. Senescent stromal cells promote cancer resistance through SIRT1 loss-potentiated overproduction of small extracellular vesicles. Cancer Res. 80, 3383-3398 (2020).

142. Patschan, S. et al. Mapping mechanisms and charting the time course of premature cell senescence and apoptosis: lysosomal dysfunction and ganglioside accumulation in endothelial cells. Am. J. Physiol. Renal Physiol. 294, F100-F109 (2008).

143. Johmura, Y. et al. Senolysis by glutaminolysis inhibition ameliorates various age-associated disorders. Science 371, 265-270 (2021).

144. Killilea, D. W., Atamna, H., Liao, C. \& Ames, B. N. Iron accumulation during cellular senescence in human fibroblasts in vitro. Antioxid Redox Signal 5, 507-516 (2003)

145. Masaldan, S. et al. Copper accumulation in senescent cells: interplay between copper transporters and impaired autophagy. Redox Biol. 16, 322-331 (2018).

146. Winter, W. E., Bazydlo, L. A. \& Harris, N. S. The molecular biology of human iron metabolism. Lab. Med. 45, 92-102 (2014).

147. Dixon, S. J. et al. Ferroptosis: an iron-dependent form of nonapoptotic cell death. Cell 149, 1060-1072 (2012).

148. Masaldan, S. et al. Iron accumulation in senescent cells is coupled with impaired ferritinophagy and inhibition of ferroptosis. Redox Biol. 14, 100-115 (2018).

149. Festa, R. A. \& Thiele, D. J. Copper: an essential metal in biology. Curr. Biol. 21, R877-R883 (2011)

150. Matos, L., Gouveia, A. \& Almeida, H. Copper ability to induce premature senescence in human fibroblasts. Age 34, 783-794 (2012).

151. Li, Y. et al. Copper induces cellular senescence in human glioblastoma multiforme cells through downregulation of Bmi-1. Oncol. Rep. 29, 1805-1810 (2013).

152. Zhang, Y. et al. A new role for oxidative stress in aging: the accelerated aging phenotype in Sod1(-/)(-) mice is correlated to increased cellular senescence. Redox Biol. 11, 30-37 (2017) 
153. Oteiza, P. I. Zinc and the modulation of redox homeostasis. Free Radic. Biol. Med. 53, 1748-1759 (2012).

154. Kumar, J. et al. Zinc levels modulate lifespan through multiple longevity pathways in Caenorhabditis elegans. PLoS ONE 11, e0153513 (2016).

155. Salazar, G., Huang, J., Feresin, R. G., Zhao, Y. \& Griendling, K. K. Zinc regulates Noxl expression through a NF-kappaB and mitochondrial ROS dependent mechanism to induce senescence of vascular smooth muscle cells. Free Radic. Biol. Med. 108, 225-235 (2017).

156. Malavolta, M. et al. Changes in $\mathrm{Zn}$ homeostasis during long term culture of primary endothelial cells and effects of $\mathrm{Zn}$ on endothelial cell senescence. Exp. Gerontol. 99, 35-45 (2017).

157. Rudolf, E. \& Cervinka, M. Stress responses of human dermal fibroblasts exposed to zinc pyrithione. Toxicol. Lett. 204, 164-173 (2011).

158. Aird, K. M. et al. Suppression of nucleotide metabolism underlies the establishment and maintenance of oncogene-induced senescence. Cell Rep. 3, 1252-1265 (2013)

159. Aird, K. M. et al. ATM couples replication stress and metabolic reprogramming during cellular senescence. Cell Rep. 11, 893-901 (2015).

160. Buj, R. et al. Suppression of p16 induces mTORC1-mediated nucleotide metabolic reprogramming. Cell Rep. 28, 1971-1980 (2019).

161. Santinon, G. et al. dNTP metabolism links mechanical cues and YAP/TAZ to cell growth and oncogene-induced senescence. EMBO J. https://doi.org/ 10.15252/embj.201797780 (2018).

162. Baar, M. P. et al. Targeted apoptosis of senescent cells restores tissue homeostasis in response to chemotoxicity and aging. Cell 169, 132-147 (2017).

163. Farr, J. N. et al. Targeting cellular senescence prevents age-related bone loss in mice. Nat. Med. 23, 1072-1079 (2017).

164. Jeon, O. H. et al. Local clearance of senescent cells attenuates the development of post-traumatic osteoarthritis and creates a pro-regenerative environment. Nat. Med. 23, 775-781 (2017).

165. Alimirah, F. et al. Cellular senescence promotes skin carcinogenesis through p38MAPK and p44/42MAPK signaling. Cancer Res. 80, 3606-3619 (2020).

166. Demaria, M. et al. Cellular senescence promotes adverse effects of chemotherapy and cancer relapse. Cancer Discov. 7, 165-176 (2017).

167. Wiley, C. D. et al. SILAC analysis reveals increased secretion of hemostasis-related factors by senescent cells. Cell Rep. 28, 3329-3337 (2019).

168. Thompson, P. J. et al. Targeted elimination of senescent beta cells prevents Type 1 diabetes. Cell Metab. 29, 1045-1060 (2019).

169. Palmer, A. K. et al. Targeting senescent cells alleviates obesity-induced metabolic dysfunction. Aging Cell 18, e12950 (2019).

170. Helman, A. et al. p16(Ink4a)-induced senescence of pancreatic beta cells enhances insulin secretion. Nat. Med. 22, 412-420 (2016).

171. Aguayo-Mazzucato, C. et al. Acceleration of beta cell aging determines diabetes and senolysis improves diabetes outcomes. Cell Metab. 30, 129-142 (2019).

172. Kim, S. R. et al. Increased renal cellular senescence in murine high-fat diet: effect of the senolytic drug quercetin. Transl Res 213, 112-123 (2019).

173. Verzola, D. et al. Accelerated senescence in the kidneys of patients with type 2 diabetic nephropathy. Am. J. Physiol. Renal Physiol. 295, F1563-F1573 (2008)

174. Crespo-Garcia, S. et al. Pathological angiogenesis in retinopathy engages cellular senescence and is amenable to therapeutic elimination via BCL-xL inhibition. Cell Metab. https://doi.org/10.1016/j.cmet.2021.01.011 (2021).

175. Heilbronn, L. K. \& Ravussin, E. Calorie restriction and aging: review of the literature and implications for studies in humans. Am. J. Clin. Nutr. 78, 361-369 (2003).

176. Fontana, L. et al. The effects of graded caloric restriction: XII. Comparison of mouse to human impact on cellular senescence in the colon. Aging Cell 17, e12746 (2018).

177. Mau, T., O'Brien, M., Ghosh, A. K., Miller, R. A. \& Yung, R. Life-span extension drug interventions affected adipose tissue inflammation in aging. J. Gerontol. A Biol. Sci. Med. Sci. 75, 89-98 (2020).

178. Cui, J. et al. Mitochondrial autophagy involving renal injury and aging is modulated by caloric intake in aged rat kidneys. PLoS ONE 8, e69720 (2013).

179. Miller, R. A. et al. Methionine-deficient diet extends mouse lifespan, slows immune and lens aging, alters glucose, T4, IGF-I and insulin levels, and increases hepatocyte MIF levels and stress resistance. Aging Cell 4, 119-125 (2005).

180. Newman, J. C. et al. Ketogenic diet reduces midlife mortality and improves memory in aging mice. Cell Metab. 26, 547-557 (2017)

181. Roberts, M. N. et al. A ketogenic diet extends longevity and healthspace in adult mice. Cell Metab. 26, 539-546e535 (2017).

182. Wang, S. Y. et al. Methionine restriction delays senescence and suppresses the senescence-associated secretory phenotype in the kidney through endogenous hydrogen sulfide. Cell Cycle 18, 1573-1587 (2019).

183. Han, Y. M. et al. beta-Hydroxybutyrate prevents vascular senescence through hnRNP A1-mediated upregulation of Oct4. Mol. Cell 71, 1064-1078 (2018).
184. Harrison, D. E. et al. Acarbose improves health and lifespan in aging HET3 mice. Aging Cell 18, e12898 (2019).

185. Chan, K. C. et al. Pleiotropic effects of acarbose on atherosclerosis development in rabbits are mediated via upregulating AMPK signals. Sci. Rep. 6, 38642 (2016).

186. Martin-Montalvo, A. et al. Metformin improves healthspan and lifespan in mice. Nat. Commun. 4, 2192 (2013).

187. Jadhav, K. S., Dungan, C. M. \& Williamson, D. L. Metformin limits ceramide-induced senescence in C2C12 myoblasts. Mech. Ageing Dev. 134, 548-559 (2013).

188. Fang, J. et al. Metformin alleviates human cellular aging by upregulating the endoplasmic reticulum glutathione peroxidase 7. Aging Cell 17, e12765 (2018).

189. Moiseeva, O. et al. Metformin inhibits the senescence-associated secretory phenotype by interfering with IKK/NF-kappaB activation. Aging Cell 12, 489-498 (2013).

190. Chen, D. et al. Metformin protects against apoptosis and senescence in nucleus pulposus cells and ameliorates disc degeneration in vivo. Cell Death Dis. 7, e2441 (2016).

191. Kim, H. et al. Metformin inhibits chronic kidney disease-induced DNA damage and senescence of mesenchymal stem cells. Aging Cell 20, e13317 (2021).

192. Simons, J. The $\$ 10$ billion pill. Fortune 147, 58-62 (2003).

193. Assmus, B. et al. HMG-CoA reductase inhibitors reduce senescence and increase proliferation of endothelial progenitor cells via regulation of cell cycle regulatory genes. Circ. Res. 92, 1049-1055 (2003).

194. Liu, S. et al. Simvastatin suppresses breast cancer cell proliferation induced by senescent cells. Sci. Rep. 5, 17895 (2015)

195. Childs, B. G. et al. Senescent intimal foam cells are deleterious at all stages of atherosclerosis. Science 354, 472-477 (2016).

196. Efimova, E. V. et al. HMG-CoA reductase inhibition delays DNA repair and promotes senescence after tumor irradiation. Mol. Cancer Ther. 17, 407-418 (2018).

197. Moon, S. H. et al. p53 Represses the mevalonate pathway to mediate tumor suppression. Cell 176, 564-580e519 (2019).

198. Garcia-Valles, R. et al. Life-long spontaneous exercise does not prolong lifespan but improves health span in mice. Longev. Healthspan. 2, 14 (2013).

199. Nilsson, M. I. et al. Lifelong aerobic exercise protects against inflammaging and cancer. PLoS ONE 14, e0210863 (2019).

200. Schafer, M. J. et al. Late-life time-restricted feeding and exercise differentially alter healthspan in obesity. Aging Cell 18, e12966 (2019).

201. Schafer, M. J. et al. Exercise prevents diet-induced cellular senescence in adipose tissue. Diabetes 65, 1606-1615 (2016).

202. Rossman, M. J. et al. Endothelial cell senescence with aging in healthy humans: prevention by habitual exercise and relation to vascular endothelial function. Am. J. Physiol. Heart. Circ. Physiol. 313, H890-H895 (2017).

203. Werner, C. et al. Physical exercise prevents cellular senescence in circulating leukocytes and in the vessel wall. Circulation 120, 2438-2447 (2009).

204. Cherasse, Y. et al. The leptomeninges produce prostaglandin D2 involved in sleep regulation in mice. Front. Cell Neurosci. 12, 357 (2018).

205. Urade, Y. \& Hayaishi, O. Prostaglandin D2 and sleep/wake regulation. Sleep Med. Rev. 15, 411-418 (2011).

206. Mander, B. A., Winer, J. R. \& Walker, M. P. Sleep and human aging. Neuron 94, 19-36 (2017).

207. Roberts, S. B. et al. Healthy aging-nutrition matters: start early and screen often. Adv. Nutr. https://doi.org/10.1093/advances/nmab032 (2021).

208. Ogrodnik, M. et al. Cellular senescence drives age-dependent hepatic steatosis. Nat. Commun. 8, 15691 (2017).

\section{Author contributions}

C.D.W. and J.C. conceived of the concepts described in this review. C.D.W. wrote the metabolic sections with assistance from J.C. J.C. wrote the introduction with assistance from C.D.W. Both authors wrote the conclusions section.

\section{Competing interests}

J.C. is a scientific founder of Unity Biotechnology, which develops senolytic therapies. C.D.W. and J.C. hold patents for induction and detection of senolysis using metabolic targets.

\section{Additional information}

Correspondence should be addressed to Christopher D. Wiley.

Peer review information Nature Metabolism thanks David Bernard and the other, anonymous, reviewer(s) for their contribution to the peer review of this work. Primary handling editor: Christoph Schmitt.

Reprints and permissions information is available at www.nature.com/reprints. Publisher's note Springer Nature remains neutral with regard to jurisdictional claims in published maps and institutional affiliations.

(c) Springer Nature Limited 2021 